\title{
All Finite Generalized Tetrahedron Groups
}

\author{
Benjamin Fine, Miriam Hahn, \\ Alexander Hulpke, Volkmar große Rebel, \\ Gerhard Rosenberger, Martin Scheer
}

Fakultät für Mathematik

Technische Universität Dortmund

Vogelpothsweg 87

44227 Dortmund
Preprint Nr. 2008-03

April 2008

$\underline{\text { tu-dortmund.de/MathPreprints }}$ 



\title{
All Finite Generalized Tetrahedron Groups
}

\author{
Benjamin Fine \\ Miriam Hahn \\ Alexander Hulpke \\ Volkmar große Rebel \\ Gerhard Rosenberger \\ Martin Scheer *
}

April 8, 2008

\begin{abstract}
A generalized tetrahedron groups is defined to be a group admitting a presentation

$$
\left\langle x, y, z \mid x^{l}=y^{m}=z^{n}=W_{1}^{p}(x, y)=W_{2}^{q}(y, z)=W_{3}^{r}(x, z)=1\right\rangle
$$

where $l, m, n, p, q, r \geq 2$, each $W_{i}(a, b)$ is a cyclically reduced word involving both $a$ and $b$. These groups appear in many contexts, not least as fundamental groups of certain hyperbolic orbifolds or as subgroups of generalized triangle groups. In this paper, we build on previous work to give a complete classification of all finite generalized tetrahedron groups.
\end{abstract}

*2000 primary 20F05; secondary 20E05, 20E07, 20H10.

Keywords: generalized tetrahedron groups, generalized triangle groups, triangle of groups, Fortsetzungssatz. 


\section{INTRODUCTION}

If $T$ is a tetrahedron in the 3 -dimensional euclidean, hyperbolic or spherical space whose dihedral angles are submultiples of $\pi$, then the reflections in the faces of $T$ generate a discrete group of isometries. The index 2 subgroup of orientation preserving isometries in this group is generated by reflections around the edges of any of the faces of $T$, and has a presentation of the form

$$
\left\langle x, y, z \mid x^{l}=y^{m}=z^{n}=\left(x y^{-1}\right)^{p}=\left(y z^{-1}\right)^{q}=\left(z x^{-1}\right)^{r}=1\right\rangle,
$$

where $l, m, n, p, q, r \geq 2$. These groups are called ordinary tetrahedron groups. Coxeter has shown in [3] and [4] that an ordinary tetrahedron group of the form 1.1 is finite if and only if the Coxeter matrix

$$
C=\left(\begin{array}{cccc}
1 & -\cos \left(\frac{\pi}{l}\right) & -\cos \left(\frac{\pi}{m}\right) & -\cos \left(\frac{\pi}{n}\right) \\
-\cos \left(\frac{\pi}{l}\right) & 1 & -\cos \left(\frac{\pi}{p}\right) & -\cos \left(\frac{\pi}{r}\right) \\
-\cos \left(\frac{\pi}{m}\right) & -\cos \left(\frac{\pi}{p}\right) & 1 & -\cos \left(\frac{\pi}{q}\right) \\
-\cos \left(\frac{\pi}{n}\right) & -\cos \left(\frac{\pi}{r}\right) & -\cos \left(\frac{\pi}{q}\right) & 1
\end{array}\right)
$$

has positive determinant. Hence, the finite ordinary tetrahedron groups are easily given.

Following Vinberg we call a group $G$ defined by a presentation

$$
G=\left\langle x, y, z \mid x^{l}=y^{m}=z^{n}=W_{1}^{p}(x, y)=W_{2}^{q}(y, z)=W_{3}^{r}(x, z)=1\right\rangle
$$

where $l, m, n, p, r \geq 2$ and each $W_{i}(a, b)$ is a cyclically reduced word involving both $a$ and $b$, a generalized tetrahedron group. For the following we may always assume that each $W_{i}(a, b)$ also is not a proper power in the free product on $a$ and $b$. If any $W_{i}(a, b)$ was a proper power it would only increase the exponent.

Certain operations on presentations of this form 1.2 do not change the groups defined by the presentations. With this in mind, we say that two presentations $P_{1}$ and $P_{2}$ of the form 1.2 are equivalent if $P_{2}$ can be obtained from $P_{1}$ by a sequence of operations of the following type:

1. Replace a generator $a$ of order $k$ by a new generator $d=a^{\alpha}$, where $\alpha$ is coprime to $k$, and then amend the relations accordingly.

2. Apply a permutation to the generators $x, y$ and $z$.

3. If $V_{i}(a, b)$ is a cyclically reduced conjugate of $W_{i}(a, b)$ in the free product on $a$ and $b$, then replace the relator $W_{i}^{k_{i}}(a, b)$ by $V_{i}^{k_{i}}(a, b)$, where $k_{i} \in$ $\{p, q, r\}$, respectively.

4. Replace the relator $W_{i}^{k_{i}}(a, b)$ by $V_{i}^{k_{i}}(a, b)$, where $V_{i}(a, b)$ is the inverse of $W_{i}(a, b)$ and $k_{i} \in\{p, q, r\}$, respectively.

5. If $a$ is a generator of order 2 , if $b$ is a generator of order $k$, if $\alpha$ and $\beta$ are coprime to $k$, and if we have a relator of the form $W=\left(a b^{\alpha}\right)^{2}$, then replace $W$ by $\left(a b^{\beta}\right)^{2}$. 
It is clear that if $P_{1}$ and $P_{2}$ are equivalent, then $P_{1}$ and $P_{2}$ define the same group. In the following we often replace a presentation of the form 1.2 by an equivalent one and work up to equivalence.

The purpose of this paper is to present the complete classification of all finite generalized tetrahedron groups.

\section{PRELIMINARY RESULTS}

For the benefit of the reader, we first present here some preliminary definitions and results that will be needed in this paper. Suppose that $G$ is defined by a presentation of the form 1.2. Let (after conjugation if necessary) $W_{1}(x, y)=$ $x^{\alpha_{1}} y^{\beta_{1}} \cdots x^{\alpha_{k_{1}}} y^{\beta_{k_{1}}}$ with $k_{1} \geq 1,1 \leq \alpha_{i}<l, 1 \leq \beta_{i}<m$ for each $i$. We similary take $k_{2}$ and $k_{3}$ to be half the length, the block length, of the cyclically reduced words $W_{2}(y, z)$ and $W_{3}(x, z)$, respectively.

$G$ can be realized as a triangle of groups, that is, as the colimit of the diagram of groups and injective homomorphisms shown in the figure

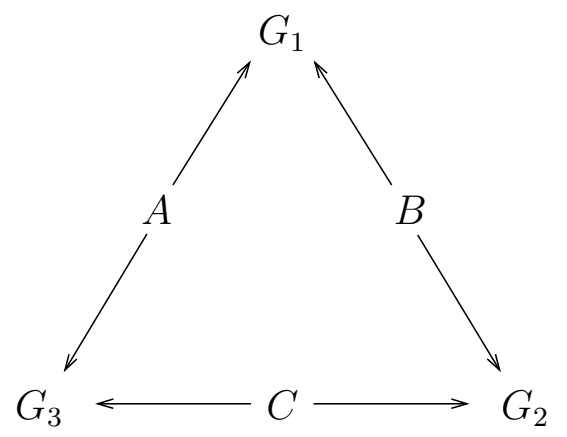

in which

$$
\begin{aligned}
& G_{1}=\left\langle x, y \mid x^{l}=y^{m}=W_{1}^{p}(x, y)=1\right\rangle, A=\left\langle x \mid x^{l}=1\right\rangle, \\
& G_{2}=\left\langle y, z \mid y^{m}=z^{n}=W_{2}^{q}(y, z)=1\right\rangle, B=\left\langle y \mid y^{m}=1\right\rangle, \\
& G_{3}=\left\langle x, z \mid x^{l}=z^{n}=W_{3}^{r}(x, z)=1\right\rangle, C=\left\langle z \mid z^{n}=1\right\rangle .
\end{aligned}
$$

We refer $G_{1}, G_{2}$ and $G_{3}$ as vertex groups and $A, B$ and $C$ as edge groups. Groups with a presentation as $G_{1}, G_{2}$ and $G_{3}$ are called generalized triangle groups.

The next theorem is a summary of the main results in [5], [6], [13] and [18]. A version which handles the Tits alternative for those groups can be found in [13].

Theorem 2.1. Let $G$ be a generalized tetrahedron group of the form 1.2 as above. If $\frac{1}{p k_{1}}+\frac{1}{q k_{2}}+\frac{1}{r k_{3}} \leq 1$ then $G$ is infinite, and if $\frac{1}{p k_{1}}+\frac{1}{q k_{2}}+\frac{1}{r k_{3}}<1$ then $G$ has a non-abelian free subgroup. 
The finite generalized tetrahedron groups of the form 1.2 with $\frac{1}{p k_{1}}+\frac{1}{q k_{2}}+\frac{1}{r k_{3}}>1$ and $(p, q, r) \neq(2,2,2)$ are classified in [5] and [17], and we refer to these papers. A more general version which handles the Tits alternative for the situation $(p, q, r) \neq(2,2,2)$ can be found in [11].

To classify the finite generalized tetrahedron groups we are left with the case $(p, q, r)=(2,2,2)$ and $\frac{1}{k_{1}}+\frac{1}{k_{2}}+\frac{1}{k_{3}}>1$. From the theorem it follows that two of the $k_{1}, k_{2}, k_{3}$ are 1 .

Hence, up to equivalence, we are left with the generalized tetrahedron groups which have a presentation of the form

$$
G=\left\langle x, y, z \mid x^{l}=y^{m}=z^{n}=W_{1}^{2}(x, y)=\left(y^{\gamma} z^{\delta}\right)^{2}=\left(x^{\alpha} z^{\beta}\right)^{2}=1\right\rangle
$$

with $W_{1}(x, y)=x^{\alpha_{1}} y^{\beta_{1}} \cdots x^{\alpha_{k}} y^{\beta_{k}}, k=k_{1} \geq 1, l, m, n \geq 2,1 \leq \alpha_{1}, \ldots, \alpha_{k}$, $\alpha<l, 1 \leq \beta_{1}, \ldots, \beta_{k}, \gamma<m$ and $1 \leq \delta, \beta<n$. We may assume $2 \leq l \leq m$, and this should hold always for a presentation of the form 2.1.

Tsaranov [21] and [22] classified the finite generalized tetrahedron groups with $k=1$. We state his result for convenience. Numerical calculations can also be found in [20].

Theorem 2.2. Let $G$ be an generalized tetrahedron group of the form 2.1 with $k=1$, which is not equivalent to a presentation for an ordinary tetrahedron group. Then, up to equivalence, $G$ is one of the following:

1. $G=\left\langle x, y, z \mid x^{3}=y^{3}=z^{3}=(x y)^{2}=(y z)^{2}=(x z)^{2}=1\right\rangle$ with $|G|=60$,

2. $G=\left\langle x, y, z \mid x^{3}=y^{3}=z^{4}=(x y)^{2}=(y z)^{2}=(x z)^{2}=1\right\rangle$ with $|G|=192$,

3. $G=\left\langle x, y, z \mid x^{3}=y^{3}=z^{5}=(x y)^{2}=(y z)^{2}=(x z)^{2}=1\right\rangle$ with $|G|=7200$,

4. $G=\left\langle x, y, z \mid x^{3}=y^{3}=z^{5}=(x y)^{2}=\left(y z^{2}\right)^{2}=(x z)^{2}=1\right\rangle$ with $|G|=7200$,

5. $G=\left\langle x, y, z \mid x^{3}=y^{3}=z^{5}=(x y)^{2}=\left(y z^{3}\right)^{2}=(x z)^{2}=1\right\rangle$ with $|G|=7200$.

There are some additional numerical restrictions for a generalized tetrahedron group of the form 2.1 to be finite.

Theorem 2.3. [6] Let $G$ be a generalized tetrahedron group of the form 2.1. If $\frac{1}{l}+\frac{1}{m} \leq \frac{1}{2}, \frac{1}{m}+\frac{1}{n} \leq \frac{1}{2}$ or $\frac{1}{l}+\frac{1}{n} \leq \frac{1}{2}$ then $G$ is infinite.

Hence, for the classification of all finite generalized tetrahedron groups of the form 2.1 we have to consider those with the additional numerical conditions that $2 \leq l \leq m, k \geq 2, \frac{1}{l}+\frac{1}{m}>\frac{1}{2}, \frac{1}{m}+\frac{1}{n}>\frac{1}{2}$ and $\frac{1}{l}+\frac{1}{n}>\frac{1}{2}$.

We also need several preliminary results about linear representations into $\operatorname{PSL}(2, \mathbb{C})$. Let $G$ be a generalized tetrahedron group given by a presentation of the form 1.2. We say that the representation $\rho: G \rightarrow \operatorname{PSL}(2, \mathbb{C})$ is essential if the elements $\rho(x), \rho(y), \rho(z), \rho\left(W_{1}(x, y)\right), \rho\left(W_{2}(y, z)\right)$ and $\rho\left(W_{3}(x, z)\right)$ have 
orders $l, m, n, p, q, r$ respectively in $\rho(G)$. In this case, we also have essential representations of the three generalized triangle groups $G_{1}, G_{2}$ and $G_{3}$ defined above as the vertex groups of the triangle of groups for $G$. In the following we use the notation $G_{1}, G_{2}$ and $G_{3}$ for these vertex groups for $G$. We use the term essential representation into $\operatorname{PSL}(2, \mathbb{C})$ analogously for $G_{1}, G_{2}, G_{3}$.

Theorem 2.4. [9] and [10] Every generalized tetrahedron group of the form 1.2 admits an essential representation in $\operatorname{PSL}(2, \mathbb{C})$.

Theorem 2.5. [9] and [10] [Fortsetzungssatz]

Let $G$ be the generalized tetrahedron group of the form 1.2, and let $G_{1}$ be the generalized triangle group defined by the presentation $G_{1}=\langle x, y| x^{l}=$ $\left.y^{m}=W_{1}^{p}(x, y)=1\right\rangle$. Suppose that $\rho_{1}$ is an essential representation of $G_{1}$ into $\operatorname{PSL}(2, \mathbb{C})$ with $X=\rho_{1}(x)$ and $Y=\rho_{1}(y)$ and one of the following two possibilities occurs:

i) $\operatorname{tr}[X, Y] \neq 2$.

ii) $(n, q, r) \neq(2,2,2)$ and $\langle X, Y\rangle$ is an infinite metabelian subgroup of $\operatorname{PSL}(2, \mathbb{C})$.

Then there is an essential representation $\rho: G \rightarrow \operatorname{PSL}(2, \mathbb{C})$ such that $X=\rho(x)$ and $Y=\rho(y)$.

Moreover, if in case $i)$ the group $\langle X, Y\rangle$ is non-elementary, then $G$ contains a non-abelian free subgroup in both cases.

Remark 2.6. A subgroup of $\operatorname{PSL}(2, \mathbb{C})$ is said to be non-elementary if it is not solvable-by-finite; such a subgroup must contain a free subgroup of rank 2 .

If $G_{1}$ is as in theorem 2.4 and $\langle X, Y\rangle$ is an abelian group other than the elementary abelian group of order 4 , then there always exists an essential representation $\sigma: G_{1} \rightarrow \operatorname{PSL}(2, \mathbb{C})$ such that $\langle\sigma(x), \sigma(y)\rangle$ is an infinite metabelian group.

It is possible to use linear representations even though each essential representation into $\operatorname{PSL}(2, \mathbb{C})$ of each of the vertex groups $G_{1}, G_{2}$ and $G_{3}$ is finite. It is possible here to have an essential representation $\rho: G \rightarrow \operatorname{PSL}(2, \mathbb{C})$ with $\rho(G)$ non-elementary. For this we use the next theorem which is straightforward.

Theorem 2.7. Let $A, B, C, D \in S L(2, \mathbb{C})$ with $A B=C$ and $D$ arbitrary.

Let $A=\left(\begin{array}{ll}a_{1} & a_{2} \\ a_{3} & a_{4}\end{array}\right), B=\left(\begin{array}{ll}b_{1} & b_{2} \\ b_{3} & b_{4}\end{array}\right), C=\left(\begin{array}{ll}c_{1} & c_{2} \\ c_{3} & c_{4}\end{array}\right), D=\left(\begin{array}{ll}d_{1} & d_{2} \\ d_{3} & d_{4}\end{array}\right)$, $\vec{d}=\left(\begin{array}{c}d_{1} \\ d_{2} \\ d_{3} \\ d_{4}\end{array}\right), \vec{r}=\left(\begin{array}{c}\operatorname{tr} D \\ \operatorname{tr} A D \\ \operatorname{tr} B D \\ \operatorname{tr} C D\end{array}\right), M=\left(\begin{array}{cccc}1 & 0 & 0 & 1 \\ a_{1} & a_{3} & a_{2} & a_{4} \\ b_{1} & b_{3} & b_{2} & b_{4} \\ c_{1} & c_{3} & c_{2} & c_{4}\end{array}\right)$.

Then $M \cdot \vec{d}=\vec{r}$ and $\operatorname{det} M=\operatorname{tr}[A, B]-2$. Moreover, if $\operatorname{det} M \neq 0$, that is $\operatorname{tr}[A, B] \neq 2$, then $\operatorname{det} D=d_{1} d_{4}-d_{2} d_{3}=1$ defines a quadratic polynomial $f(t) \in K[t], K=\mathbb{Q}\left(a_{1}, a_{2}, a_{3}, a_{4}, b_{1}, b_{2}, b_{3}, b_{4}, \operatorname{tr} D, \operatorname{tr} A D, \operatorname{tr} B D\right)$ with highest coefficient $\alpha_{2}=-\frac{1}{\operatorname{det} M}$ and zeros $\operatorname{tr} A B D$ and $\operatorname{tr} B A D$. 
This theorem may be used in the following manner. Let $G$ be a generalized tetrahedron group of the form 1.2 with vertex groups $G_{1}, G_{2}$ and $G_{3}$ as above. Let $\rho_{1}: G_{1} \rightarrow \operatorname{PSL}(2, \mathbb{C}), \rho_{1}(x)=X, \rho_{1}(y)=Y$, be an essential representation of $G_{1}$ into $\operatorname{PSL}(2, \mathbb{C})$. Let $\langle X, Y\rangle$ be non-cyclic and finite, that is, $\langle X, Y\rangle$ is isomorphic to a dihedral group $D_{2 n}, n \geq 2, A_{4}, S_{4}$ or $A_{5}$. We remark that $\operatorname{tr}[X, Y] \neq 2$. Then we use Theorem 2.7 to construct $Z$ to get an essential representation $\rho: G \rightarrow \operatorname{PSL}(2, \mathbb{C})$ with $\rho(x)=X, \rho(y)=Y$ and $\rho(z)=Z$. If $X Y Z$ has infinite order, then $\langle X, Y, Z\rangle$ is non-elementary, and hence, $G$ has a non-abelian free subgroup.

\section{THE FINAL CLASSIFICATION OF THE FI- NITE GENERALALIZED TETRAHEDRON GROUPS}

As pointed out in section 2 in order to get the final classification of the finite generalized tetrahedron groups we have to consider the groups defined by a presentation

$$
G=\left\langle x, y, z \mid x^{l}=y^{m}=z^{n}=W_{1}^{2}(x, y)=\left(y^{\gamma} z^{\delta}\right)^{2}=\left(x^{\alpha} z^{\beta}\right)^{2}=1\right\rangle
$$

with $W_{1}(x, y)=x^{\alpha_{1}} y^{\beta_{1}} \cdots x^{\alpha_{k}} y^{\beta_{k}}, k \geq 2,2 \leq l \leq m, 2 \leq n, 1 \leq \alpha_{1}, \ldots, \alpha_{k}$, $\alpha<l, 1 \leq \beta_{1}, \ldots, \beta_{k}, \gamma<m, 1 \leq \delta, \beta<n, \frac{1}{l}+\frac{1}{m}>\frac{1}{2}, \frac{1}{l}+\frac{1}{n}>\frac{1}{2}$ and $\frac{1}{m}+\frac{1}{n}>\frac{1}{2}$. As already mentioned we assume that $W_{1}(x, y)$ ist not a proper power in the free product on $a$ and $b$.

Theorem 3.1. Let $G$ be a generalized tetrahedron group of the form 3.1 together with the given numerical conditions. Let $W_{1}(x, y)$ be not a proper power in the free product on $a$ and $b$. If $G$ is finite then, up to equivalence, $G$ is one of the following:

1. $\left\langle x, y, z \mid x^{2}=y^{3}=z^{2}=\left(x y x y^{2}\right)^{2}=(y z)^{2}=(x z)^{2}=1\right\rangle$ of order 48 ,

2. $\left\langle x, y, z \mid x^{2}=y^{3}=z^{3}=\left(x y x y^{2}\right)^{2}=(y z)^{2}=(x z)^{2}=1\right\rangle$ of order 120 ,

3. $\left\langle x, y, z \mid x^{2}=y^{3}=z^{4}=\left(x y x y^{2}\right)^{2}=(y z)^{2}=(x z)^{2}=1\right\rangle$ of order 384 ,

4. $\left\langle x, y, z \mid x^{2}=y^{3}=z^{5}=\left(x y x y^{2}\right)^{2}=(y z)=(x z)^{2}=1\right\rangle$ of order 14400,

5. $\left\langle x, y, z \mid x^{2}=y^{3}=z^{2}=\left(x y x y x y^{2}\right)^{2}=(y z)^{2}=(x z)^{2}=1\right\rangle$ of order 96 ,

6. $\left\langle x, y, z \mid x^{2}=y^{3}=z^{3}=\left(x y x y x y^{2}\right)^{2}=(y z)^{2}=(x z)^{2}=1\right\rangle$ of order 384,

7. $\left.\langle x, y, z| x^{2}=y^{3}=z^{2}=\left(\text { xyxyxyxy } y^{2}\right)^{2}=(y z)^{2}=(x z)^{2}=1\right\rangle$ of order 240 ,

8. $\left\langle x, y, z \mid x^{2}=y^{3}=z^{3}=\left(x y x y x y x y^{2}\right)^{2}=(y z)^{2}=(x z)^{2}=1\right\rangle$ of order 14400, 
9. $\left\langle x, y, z \mid x^{2}=y^{3}=z^{2}=\left(x y x y x y^{2} x y^{2}\right)=(y z)^{2}=(x z)^{2}=1\right\rangle$ of order 1152 ,

10. $\left\langle x, y, z \mid x^{2}=y^{3}=z^{3}=\left(x y x y x y^{2} x y^{2}\right)^{2}=(y z)^{2}=(x z)^{2}=1\right\rangle$ of order 23040 ,

11. $\left\langle x, y, z \mid x^{2}=y^{3}=z^{2}=\left(x y x y x y^{2} x y x y^{2}\right)^{2}=(y z)^{2}=(x z)^{2}=1\right\rangle$ of order 1440 ,

12. $\left\langle x, y, z \mid x^{2}=y^{3}=z^{3}=\left(x y x y x y^{2} x y x y^{2}\right)^{2}=(y z)^{2}=(x z)^{2}=1\right\rangle$ of order 345600 ,

13. $\left.\langle x, y, z| x^{2}=y^{3}=z^{2}=\left(\text { xyxyxyxy } y^{-1} x y^{-1}\right)^{2}=(y z)^{2}=(x z)^{2}=1\right\rangle$ of order 5760 ,

14. $\left.\langle x, y, z| x^{2}=y^{3}=z^{3}=\left(\text { xyxyxyxy } y^{-1} x y^{-1}\right)^{2}=(y z)^{2}=(x z)^{2}=1\right\rangle$ of order 2764800 ,

15. $\left\langle x, y, z \mid x^{2}=y^{3}=z^{2}=\left(x y x y x y^{2} x y^{2} x y x y^{2}\right)^{2}=(y z)^{2}=(x z)^{2}=1\right\rangle$ of order 5760 ,

16. $\left.\langle x, y, z| x^{2}=y^{3}=z^{2}=\left(\text { xyxyxyxy } y^{2} x y x y^{2} x y^{2}\right)^{2}=(y z)^{2}=(x z)^{2}=1\right\rangle$ of order 11520 ,

17. $\langle x, y, z| x^{2}=y^{3}=z^{2}=\left(x y x y x y x y^{2} x y^{2} x y x y^{2} x y^{2}\right)^{2}=(y z)^{2}$ $\left.=(x z)^{2}=1\right\rangle$ of order 849346560 ,

18. $\left\langle x, y, z \mid x^{2}=y^{4}=z^{2}=\left(x y x y x y^{3}\right)^{2}=(y z)^{2}=(x z)^{2}=1\right\rangle$ of order 384,

19. $\left\langle x, y, z \mid x^{2}=y^{4}=z^{3}=\left(x y x y x y^{3}\right)^{2}=(y z)^{2}=(x z)^{2}=1\right\rangle$ of order 9216,

20. $\left\langle x, y, z \mid x^{2}=y^{5}=z^{2}=\left(x y x y^{2}\right)^{2}=(y z)^{2}=(x z)^{2}=1\right\rangle$ of order 240,

21. $\left\langle x, y, z \mid x^{2}=y^{5}=z^{3}=\left(x y x y^{2}\right)^{2}=\left(y^{2} z\right)^{2}=(x z)^{2}=1\right\rangle$ of order 14400,

22. $\left\langle x, y, z \mid x^{2}=y^{5}=z^{2}=\left(x y x y x y^{4}\right)^{2}=(y z)^{2}=(x z)^{2}=1\right\rangle$ of order 2400,

23. $\left\langle x, y, z \mid x^{2}=y^{5}=z^{2}=\left(x y x y^{2} x y^{3}\right)^{2}=(y z)^{2}=(x z)^{2}=1\right\rangle$ of order 2400,

24. $\left\langle x, y, z \mid x^{2}=y^{5}=z^{3}=\left(x y x y^{2} x y^{3}\right)^{2}=\left(y^{2} z\right)^{2}=(x z)^{2}=1\right\rangle$ of order 1728000 ,

25. $\left\langle x, y, z \mid x^{2}=y^{5}=z^{3}=\left(x y x y x y^{4}\right)^{2}=(y z)^{2}=(x z)^{2}=1\right\rangle$ of order 1728000 ,

26. $\left\langle x, y, z \mid x^{3}=y^{3}=z^{2}=\left(x y x^{2} y^{2}\right)^{2}=(y z)^{2}=(x z)^{2}=1\right\rangle$ of order 576,

27. $\left\langle x, y, z \mid x^{3}=y^{3}=z^{2}=\left(x y x y^{2}\right)^{2}=(y z)^{2}=(x z)^{2}=1\right\rangle$ of order 360,

28. $\left\langle x, y, z \mid x^{3}=y^{3}=z^{3}=\left(x y x^{2} y^{2}\right)^{2}=(y z)^{2}=(x z)^{2}=1\right\rangle$ of order 11520,

29. $\left\langle x, y, z \mid x^{3}=y^{3}=z^{3}=\left(x y x y^{2}\right)^{2}=(y z)^{2}=(x z)^{2}=1\right\rangle$ of order 7200 , 
30. $\left\langle x, y, z \mid x^{3}=y^{3}=z^{2}=\left(x y x y x^{-1} y^{-1}\right)^{2}=(y z)^{2}=(x z)^{2}=1\right\rangle$ of order 2880 ,

31. $\left\langle x, y, z \mid x^{3}=y^{5}=z^{2}=\left(x y x^{2} y^{2}\right)^{2}=\left(y^{2} z\right)^{2}=(x z)^{2}=1\right\rangle$ of order 43200 ,

32. $\left\langle x, y, z \mid x^{3}=y^{5}=z^{2}=\left(x^{-1} y x y^{-1} x y^{-1}\right)^{2}=(y z)^{2}=(x z)^{2}=1\right\rangle$ of order 1728000 .

Remark 3.2. In [18] we gave a provisional list for the finite generalized tetrahedron groups of the form 3.1 with $k \geq 2$. In the list in [18], because of equivalence, the groups 22. and 32. are superfluous, and for typing reasons we unfortunately gave the wrong orders for the groups 29., 30., 31. and 34. This is corrected in theorem 3.1.

Proof of theorem 3.1.

The proof consists of a complicated case by case analysis. We first observe the following. Let $\rho_{1}: G_{1} \rightarrow \operatorname{PSL}(2, \mathbb{C})$ be an essential representation with

$$
\begin{aligned}
& \rho_{1}(x)=X, \operatorname{tr} X=2 \cos \left(\frac{\pi}{l}\right) \quad \text { and } \\
& \rho_{2}(y)=Y, \operatorname{tr} Y=2 \cos \left(\frac{\pi}{m}\right) .
\end{aligned}
$$

Let $t=\operatorname{tr} X Y$. Then $f(t)=\operatorname{tr} W_{1}(X, Y)$ is a polynomial of degree $k$ over $\mathbb{Z}\left(\cos \left(\frac{\pi}{l}\right), \cos \left(\frac{\pi}{m}\right)\right)$. We call $f(t)$ the trace polynomial of $W_{1}(X, Y)$. Recall that this is only defined if $\operatorname{tr} X=2 \cos \left(\frac{\pi}{l}\right)$ and $\operatorname{tr} Y=2 \cos \left(\frac{\pi}{m}\right)$. If $f(t)$ has a multiple root then $G$ is infinite (see [18]).

From now on, let $k \geq 2,2 \leq l \leq m, \frac{1}{l}+\frac{1}{m}>\frac{1}{2}, \frac{1}{m}+\frac{1}{n}>\frac{1}{2}$ and $\frac{1}{l}+\frac{1}{n}>\frac{1}{2}$.

Since $k \geq 2$ and $W_{1}(X, Y)$ is not a proper power we have $m \geq 3$. First we prove the following

Lemma 3.3. If $m \geq 6$ then $G$ is infinite.

Proof of Lemma 3.3.

Let $m \geq 6$. First of all we must have $l=n=2$. Then $\alpha=\beta=\delta=1$. We may assume that $\gamma \mid m$.

i) Assume that $G_{1}$ admits an infinite metabelian image in $\operatorname{PSL}(2, \mathbb{C})$ via $\rho_{1}$. If $\gamma \geq 2$ we introduce the relation $y^{\gamma}=1$ to get an infinite image of $G$ (see [10], Theorem 9.2.3).

If $\gamma=1$ then the subgroup $\bar{G}_{1}=\langle x, y\rangle$ of $G$ has a presentation

$\bar{G}_{1}=\left\langle x, y \mid x^{2}=y^{m}=W_{1}^{2}(x, y)=W_{1}^{2}\left(x^{-1}, y^{-1}\right)=1\right\rangle$, and $G$ is infinite since $\rho_{1}$ factorizes via $\bar{G}_{1}$ :

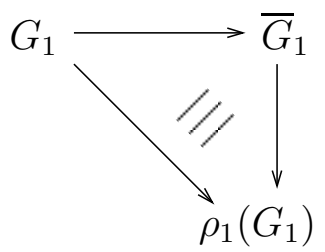


ii) So assume now that $G_{1}$ has no infinite metabelian image. Then $G$ is infinite by theorem 2.5, or the trace polynomial of $W_{1}(X, Y)$ must have a multiple zero because $k \geq 2$, and so $G$ also here is infinite.

This proves Lemma 3.3.

One argument in the proof of Lemma 3.3 can be extended.

Lemma 3.4. Assume that $G_{1}$ has an essential representation $\rho_{1}: G_{1} \rightarrow \operatorname{PSL}(2, \mathbb{C})$ with $\rho_{1}\left(G_{1}\right)$ cyclic or infinite metabelian. Then $G$ is infinite.

Proof of Lemma 3.4 .

We may assume that $\rho_{1}\left(G_{1}\right)$ is infinte metabelian. If $n \geq 3$ then $G$ is infinite by theorem 2.5.

Now, let $n=2$. Then $G$ has the factor group

$\bar{G}=\left\langle x, y, z \mid x^{l}=y^{m}=z^{2}=W_{1}^{2}(x, y)=(y z)^{2}=(x z)^{2}=1\right\rangle$ since in a dihedral group $\left\langle a, b \mid a^{2}=b^{s}=(a b)^{2}=1\right\rangle, s \geq 3$, the relation $\left(a b^{\epsilon}\right)^{2}=1,1 \leq \varepsilon<s$, is a consequence of the relation $(a b)^{2}=1$, together with $a^{2}=b^{s}=1$. The subgroup $\bar{G}_{1}=\langle x, y\rangle$ of $\bar{G}$ has a presentation $\bar{G}_{1}=\langle x, y| x^{2}=y^{m}=W_{1}^{2}(x, y)=$ $\left.W_{1}^{2}\left(x^{-1}, y^{-1}\right)=1\right\rangle$, and $G$ is infinite since $\rho_{1}$ factorizes via $\bar{G}_{1}$ :

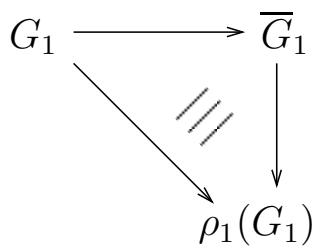

This proves Lemma 3.4.

Agreement:

From now on we assume that there is no essential representation $\rho_{1}: G_{1} \rightarrow \operatorname{PSL}(2, \mathbb{C})$ with $\rho\left(G_{1}\right)$ cyclic or infinite metabelian.

From these lemmas and the above inequalities we get $2 \leq l, n \leq 5$ and $3 \leq m \leq 5$. Our strategy is to make case by case considerations for the cases $(l, m)=(2,3),(2,4),(2,5),(3,3),(3,4)$ and $(3,5)$. Before we do that we describe a computational method using GAP which covers many groups which occur in the subsequent considerations. 


\section{A. Computational method using GAP}

A standard method for proving the infiniteness of a finitely presented group $G$ computationally is to find a subgroup $U<G$ of finite index such that $U / U^{\prime}$ (the structure of which can be obtained by Reidmeister-Schreier rewriting and a Smith normal form computation, see section 9.3.3 in [7]) has an infinite cyclic factor. Here U' means the commutator subgroup of $U$.

As it is not clear a priori whether such subgroups exist with a sufficiently small index $[G: U]$ to enable such a computation the traditional approach for this has been to calculate subgroups using a low-index algorithm (see section 5.4 in [7]). The exponential complexity of this algorithm however typically restricts this to an index substantially smaller than 100 . Often the only subgroups with infinite abelianization have larger index.

If this happens we can use the result of a limited low-index calculation to obtain further subgroups, sometimes of substantially larger index:

The simultaneous action of $G$ on the cosets of several subgroups (found via lowindex calculations) yields an intransitive permutation representation $\varphi: G \rightarrow$ $P<S_{n}$ for $n$ the sum of the indices of the subgroups. Each subgroup $S$ of $P$ yields as its preimage $U:=\varphi^{-1}(S)$ a subgroup of $G$ with $[P: S]=[G: U]$.

As $P$ is a permutation group we can use the rich pool of permutation group algorithms, in particular (iterative) calculation of maximal subgroups (see [1] and [8]), to determine further subgroups $U$ of $G$ of larger index than feasible using a low-index calculation. Furthermore, even if $\left[U: U^{\prime}\right]$ is finite we can induce the representation homomorphismus $\psi: U \rightarrow U / U^{\prime}$ to a (permutation) representation of $G$ [15], exposing an even larger quotient to search in for subgroups $S$. This process can be iterated.

We illustrate this procedure for two of the groups we considered with this method:

1. Let $G=\langle x, y, z| x^{3}=y^{5}=z^{3}=\left(x y^{2} x^{2} y^{2} x y x^{2} y\right)^{2}=\left(y^{4} z\right)^{2}$ $\left.=(x z)^{2}=1\right\rangle$. A low-index calculation finds a subgroup of index 12 such that the action on the cosets yields the permutation presentation:

$$
\begin{aligned}
& x \quad \longmapsto \\
& y \quad(1,2,4)(3,6,11)(5,7,8)(9,10,12), \\
& z \quad(2,5,10,7,3)(4,8,11,12,9), \\
& z \quad(1,3,7)(2,5,10)(4,9,6)(8,11,12)
\end{aligned}
$$

whose image is the Mathieu group $M_{12}$. This group has (up to conjugacy) a maximal subgroup $S$ of index 144. Its pre-image $U=\varphi^{-1}(S)$ has an infinite cyclic factor and thus is finite. We will cover this example simultaneously with other groups later in this article, using another method.

2. For an example of a more complicated situation let

$G=\langle x, y, z| x^{2}=y^{3}=z^{3}=\left(\text { xyxyxyx } y^{2} x y x y^{2} x y^{2}\right)^{2}=(y z)^{2}=$

$\left.(x z)^{2}=1\right\rangle$. A low-index calculation in $G$ yields 21 classes of subgroups 
on index 12. The action on the cosets of a representative of one of these classes (class 16 in the arrangement given by GAP) yields the following permutation images:

$$
\begin{aligned}
& x \longmapsto(3,8)(4,5)(6,7)(10,12), \\
& y \longmapsto(1,2,3)(4,9,10)(5,11,6)(7,12,8) \text {, } \\
& z \longmapsto(1,4,5)(2,6,7)(3,8,9)(10,12,11) \text {. }
\end{aligned}
$$

In the permutation image of order 15552 we find a subgroup

$$
S=\langle(1,5)(3,12)(6,7)(8,10),(1,6,12)(3,8,4,10,7,5)(9,11)\rangle
$$

of index 48.

Let $U<G$ be the pre-image of this subgroup. We calculate that $U / U^{\prime} \cong C_{2}{ }^{4}$. Of the 15 subgroups of index 2 in $U$ (only) one has commutator factor group of type $2^{3} 3^{2}$.

Let $V<U$ be the corresponding subgroup of index 9 .

Then $V / V^{\prime} \cong \mathbb{Z}^{2} \times{C_{2}}^{3} \times{C_{3}}^{2}$, thus $V$ (and thus $G$ ) is infinite. The following sequence of GAP commands (see [12]) can be used to verify these steps.

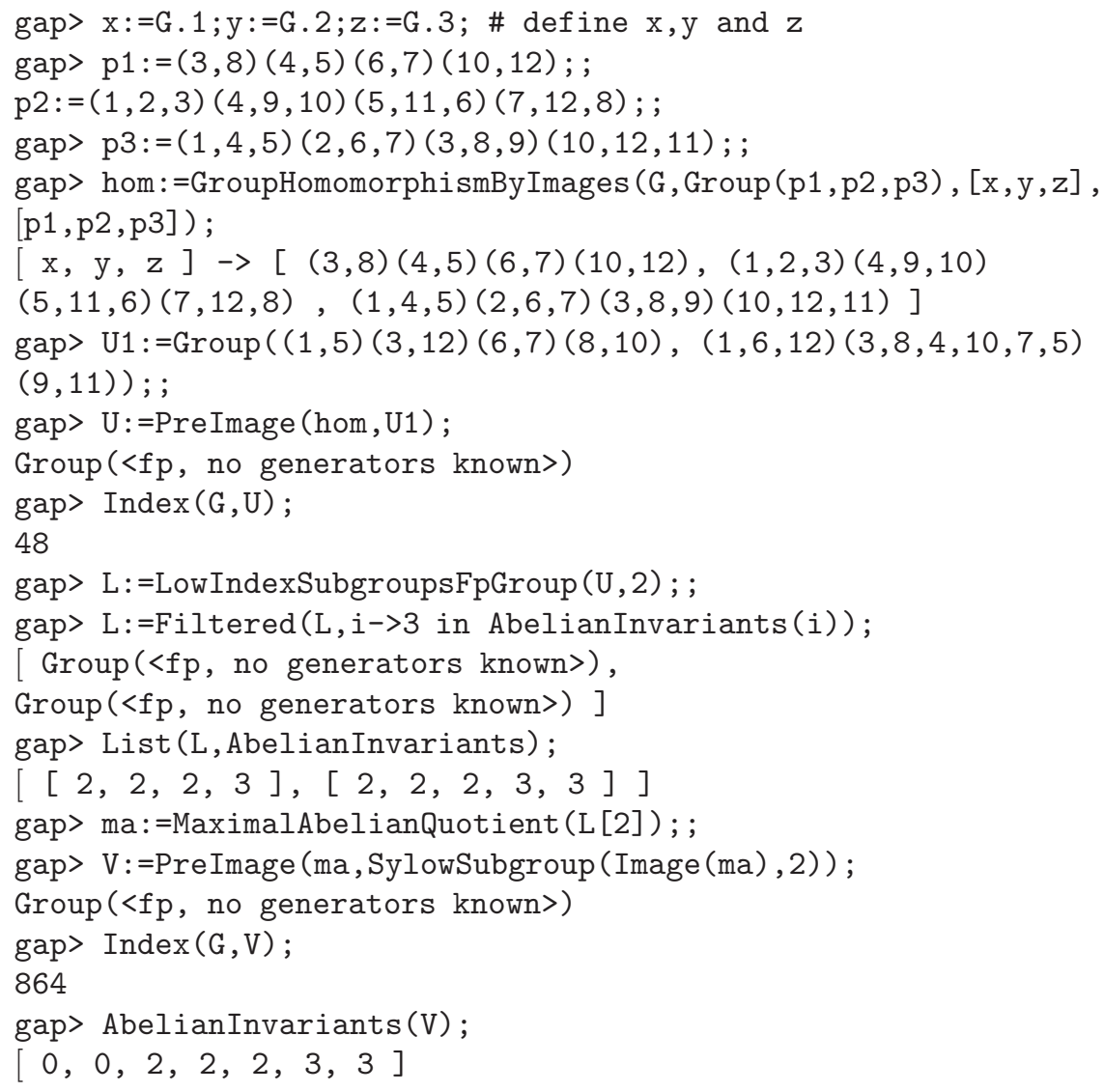


In the following case-by-case considerations we used this type of calculation in GAP extensively. Whenever we found a subgroup $U$ with $U / U^{\prime}$ infinite, we

list the following data: The index of $U$, the factors of $U / U^{\prime}$ (with a 0 indicating a factor $\mathbb{Z}$ ) and the permutation representation of $G$ on the cosets of $U$. (With this representation $U$ can be reconstructed to verify the structure of $U / U^{\prime}$.)

If the group is finite, we verified this using coset enumeration by a cyclic subgroup. In this case we will simply state the order of the group.

\section{B. Computational case by case considerations}

\subsection{Case $(1, \mathrm{~m})=(2,3)$}

\subsection{1 $k=2$ :}

Then, up to equivalence, $W_{1}(x, y)=x y x y^{2}$.

If $n=2$ then $|G|=48$.

If $n=3$ then $|G|=120$.

Now let $n=4$.

If $\beta=2$ and $\delta \neq 2$ then we introduce the relation $z^{2}=1$ to get an infinite factor group.

If $\delta=2$ then $G_{2}=\left\langle y, z \mid y^{3}=z^{4}=\left(y z^{2}\right)^{2}=1\right\rangle$ which has an essential representation $\rho_{2}: G_{2} \rightarrow \operatorname{PSL}(2, \mathbb{C})$ with $\rho_{2}\left(G_{2}\right)$ non-elementary, and hence $G$ is infinite by Fortsetzungssatz (2.5).

Now we may assume that $\delta=\beta=1$ and also $\gamma=1$, up to equivalence. Then $|G|=384$.

Now let $n=5$. Up to equivalence, we may assume that $\delta=\gamma=1$ and $1 \leq \beta \leq 2$ because $x^{2}=1$. By the dihedral equivalence relation we may in fact also assume that $\beta=1$. Then $|G|=14400$.

\subsection{2 $k=3$ :}

Then, up to equivalence, $W_{1}(x, y)=x y x y x y^{2}$.

If $n \geq 4$ then we introduce the relation $(x y)^{4}=1$ and get the factor group

$$
\bar{G}=\left\langle x, y, z \mid x^{2}=y^{3}=z^{n}=(x y)^{4}=\left(y^{\gamma} z^{\delta}\right)^{2}=\left(x z^{\beta}\right)^{2}=1\right\rangle
$$

which is infinite (see [5] and [6]).

Now let $2 \leq n \leq 3$. Then we may assume that $\gamma=\delta=\beta=1$.

If $n=2$ then $|G|=96$.

If $n=3$ then $|G|=384$.

3.1.3 $k=4$ :

Then, up to equivalence,

i) $W_{1}(x, y)=x y x y x y x y^{2}$ or 
ii) $W_{1}(x, y)=x y x y x y^{2} x y^{2}$

(recall that $W_{1}(x, y)$ is not a proper power).

i) Let first $W_{1}(x, y)=x y x y x y x y^{2}$.

If $n \geq 4$ then we introduce the relation $(x y)^{5}=1$ and get the factor group

$$
\bar{G}=\left\langle x, y, z \mid x^{2}=y^{3}=z^{n}=(x y)^{5}=\left(y^{\gamma} z^{\delta}\right)^{2}=\left(x z^{\beta}\right)^{2}=1\right\rangle
$$

which is infinite (see [5] and [6]).

Now let $2 \leq n \leq 3$. Then we may assume that $\gamma=\delta=\beta=1$.

If $n=2$ then $|G|=240$.

If $n=3$ then $|G|=14400$.

ii) Now let $W_{1}(x, y)=x y x y x y^{2} x y^{2}$.

If $n \geq 4$ then we introduce the relation $(x y)^{4}=1$ and get the factor group

$$
\bar{G}=\left\langle x, y, z \mid x^{2}=y^{3}=z^{n}=(x y)^{4}=\left(y^{\gamma} z^{\delta}\right)^{2}=\left(x z^{\beta}\right)^{2}=1\right\rangle
$$

which is infinite (see [5] and [6]).

Now let $2 \leq n \leq 3$. Then again we may assume that $\gamma=\delta=\beta=1$.

If $n=2$ then $|G|=576$.

If $n=3$ then $|G|=23040$.

\subsection{4 $k \geq 5$ :}

If $G_{1}$ has an essential representation $\rho_{1}: G_{1} \rightarrow \operatorname{PSL}(2, \mathbb{C})$ with $\rho_{1}\left(G_{1}\right)$ cyclic then $G$ is infinite. If the trace polynomial for $W_{1}(x, y)$ has a multiple root then $G$ is infinite (see [18]).

Hence, by [2] and [13] we have to consider only the following cases, up to equivalence:

i) $k=5, W_{1}(x, y)=x y x y x y^{2} x y x y^{2}$,

ii) $k=6, W_{1}(x, y)=$ xyxyxyxy $x y^{-1} x y^{-1}$,

iii) $k=6, W_{1}(x, y)=x y x y x y^{2} x y^{2} x y x y^{2}$,

iv) $k=7, W_{1}(x, y)=x y x y x y x y^{2} x y x y^{2} x y^{2}$,

v) $k=8, W_{1}(x, y)=x y x y x y x y^{2} x y^{2} x y x y^{2} x y^{2}$.

i) $k=5, W_{1}(x, y)=x y x y x y^{2} x y x y^{2}$.

If $n \geq 4$ then we introduce the relation $(x y)^{4}=1$ and get the factor group

$$
\bar{G}=\left\langle x, y, z \mid x^{2}=y^{3}=z^{n}=(x y)^{4}=\left(y^{\gamma} z^{\delta}\right)^{2}=\left(x z^{\beta}\right)^{2}=1\right\rangle
$$

which is infinite (see [5] and [6]).

Now let $2 \leq n \leq 3$. Then again we may assume $\gamma=\delta=\beta=1$.

If $n=2$ then $|\bar{G}|=1440$.

If $n=3$ then $|G|=345600$. 
ii) $k=6, W_{1}(x, y)=\operatorname{xyxyxyxy} x y^{-1} x y^{-1}$.

If $n \geq 4$ we may again introduce the relation $(x y)^{4}=1$ and get an infinite factor group.

Now let $2 \leq n \leq 3$. Then we may assume again $\gamma=\delta=\beta=1$.

If $n=2$ then $|G|=5760$.

If $n=3$ then $|G|=2764800$.

iii) $k=6, W_{1}(x, y)=x y x y x y^{2} x y^{2} x y x y^{2}$.

If $n \geq 4$ we may introduce the relation $(x y)^{5}=1$ and get again an infinite factor group.

Now let $2 \leq n \leq 3$. Then we may assume again $\gamma=\delta=\beta=1$.

If $n=2$ then $|G|=5760$.

If $n=3$ then $G$ is infinite. Here we used GAP and got the compuational data:

$72[0]$ and

$$
\begin{array}{cl}
x \longmapsto \quad(1,2) & (3,7)(4,9)(5,10)(6,12)(11,20)(13,23)(14,25)(16,29) \\
& (17,22)(18,24)(19,31)(21,28)(26,38)(27,37)(30,34) \\
& (32,42)(33,41)(35,43)(36,45)(39,47)(40,48)(44,51) \\
& (46,53)(49,57)(50,58)(52,59)(54,60)(55,61)(56,62) \\
& (63,65)(64,66)(67,69)(68,72)(70,71), \\
y \quad & (1,3,4)(2,5,6)(7,13,14)(9,16,17)(10,18,19)(12,21,22) \\
& (15,27,28)(20,33,29)(23,34,32)(24,30,26)(25,35,36) \\
& (31,39,40)(37,46,42)(38,41,44)(43,49,50)(45,52,51) \\
& (47,54,55)(48,56,53)(58,62,63)(59,64,61)(65,67,68) \\
& (66,69,70), \\
z \quad \longmapsto \quad(3,4 & 8)(5,6,11)(7,15,9)(10,20,12)(13,17,24)(14,26,27) \\
& (16,28,30)(18,22,23)(19,32,33)(21,29,34)(25,37,38) \\
& (31,41,42)(35,44,40)(36,39,46)(43,48,51)(45,53,47) \\
& (49,52,55)(50,54,56)(57,61,59)(58,62,60)(67,68,71) \\
& (69,70,72) .
\end{array}
$$

iv) $k=7, W_{1}(x, y)=x y x y x y x y^{2} x y x y^{2} x y^{2}$.

If $n \geq 4$ we may again introduce the relation $(x y)^{5}=1$ and get an infinite factor group.

Now let $2 \leq n \leq 3$ and assume again $\gamma=\delta=\beta=1$.

If $n=2$ then $|G|=11520$.

If $n=3$ then $G=\langle x, y, z| x^{2}=y^{3}=z^{3}=\left(\text { xyxyxyxy } y^{2} x y x y^{2} x y^{2}\right)^{2}=$ $\left.(y z)^{2}=(x z)^{2}=1\right\rangle$.

This is the second example in part A where we described the computational method using GAP and we showed that $G$ is infinite.

v) $k=8, W_{1}(x, y)=x y x y x y x y^{2} x y^{2} x y x y^{2} x y^{2}$

If $n \geq 4$ we may again introduce the relation $(x y)^{5}$ and get an infinite factor group.

Now let $2 \leq n \leq 3$ and assume again $\gamma=\delta=\beta=1$.

If $n=2$ then $|\bar{G}|=849346560$.

If $n=3$ then $G=\langle x, y, z| x^{2}=y^{3}=z^{3}=\left(x y x y x y x y^{2} x y^{2} x y x y^{2} x y^{2}\right)^{2}=$ 
$\left.(y z)^{2}=(x z)^{2}=1\right\rangle$, and $G$ is infinite. Here we used GAP and got that $G$ has a subgroup $U$ of index 18 with $U / U^{\prime}$ infinite via the permutation representation

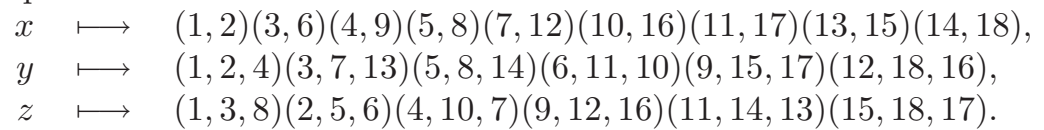

The factors of $U / U^{\prime}$ are $[0,2,4]$.

\subsection{Case $(1, \mathrm{~m})=(2,4)$}

We have $2 \leq n \leq 3$ because $\frac{1}{m}+\frac{1}{n}>\frac{1}{2}$. Then we may assume $\beta=\delta=1$ because $l=2$. If $G_{1}$ has an essential representation $\rho_{1}: G_{1} \rightarrow \operatorname{PSL}(2, \mathbb{C})$ with $\rho_{1}\left(G_{1}\right)$ non-elementary then $G$ is infinite by Fortsetzungssatz $(2.5)$.

If $G_{1}$ has an essential representation $\rho_{1}: G_{1} \rightarrow \operatorname{PSL}(2, \mathbb{C})$ with $\rho_{1}\left(G_{1}\right)$ cyclic or infinite metabelian and $n \geq 3$ then $G$ is infinite by Fortsetzungssatz (2.5) and the remark after the theorem.

If the trace polynomial of $W_{1}(x, y)$ has a multiple root then $G$ is infinite (see [18]).

If $n=3$ and $G_{2}=\left\langle y, z \mid y^{4}=z^{3}=\left(z y^{2}\right)^{2}=1\right\rangle$ then $G_{2}$ has an essential representation $\rho_{2}: G_{2} \rightarrow \operatorname{PSL}(2, \mathbb{C})$ with $\rho_{2}\left(G_{2}\right)$ non-elementary and hence $G$ is infinite by Fortsetzungssatz (2.5).

We always have this in mind.

\subsection{1 $k=2$ :}

Up to equivalence, we have consider the following two cases:

i) $W_{1}(x, y)=x y x y^{3}$,

ii) $W_{1}(x, y)=x y x y^{2}$.

We consider the two cases in a parallel manner. We may assume that $\beta=\delta=1$. Let first $\gamma=1$. We consider the subgroup $H$ generated by $x_{1}=y, x_{2}=x y x$ and $x_{3}=z . H$ has index 2 in $G$ and a presentation

$H=\left\langle x_{1}, x_{2}, x_{3} \mid x_{1}^{4}=x_{2}^{4}=x_{3}^{n}=\left(x_{1} x_{2}^{\varepsilon}\right)^{2}=\left(x_{2} x_{1}^{\varepsilon}\right)^{2}=\left(x_{1} x_{3}\right)^{2}=\left(x_{2} x_{3}^{-1}\right)^{2}=1\right\rangle$

with $\varepsilon=2$ or $3, n=2$ or 3 .

If $\varepsilon=2$ we introduce the relations $x_{1}^{2}=x_{2}^{2}$ and get the infinite factor group

$$
\bar{H}=\left\langle x_{1}, x_{2}, x_{3} \mid x_{1}^{2}=x_{2}^{2}=x_{3}^{n}=\left(x_{1} x_{3}\right)^{2}=\left(x_{2} x_{3}\right)^{2}=1\right\rangle .
$$

If $\varepsilon=3$ then the relation $\left(x_{2} x_{1}^{3}\right)^{2}=1$ is superfluous because $\left(x_{2} x_{1}^{3}\right)^{-1}=x_{1} x_{2}^{3}$, and $H$ is infinite by theorem 2.2 .

Now, let $2 \leq \gamma \leq 3$. Since $\left(y^{3} z^{\delta}\right)^{-1}=z^{-\delta} y$ the case $\gamma=3$ follows from the case $\gamma=1$ when we replace $z$ by $z^{-1}$ if necessary. Hence, we may assume that $\gamma=2$.

If $n=3$ then $G$ is infinite by the above remark.

If $n=2$ then we introduce the relation $y^{2}=1$ and get an infinite factor group. 


\subsection{2 $k=3$ :}

If $n=3$ and $\gamma=2$ then $G$ is infinite by the above remarks.

Hence, let $\gamma \neq 2$ if $n=3$.

By [16], up to equivalence, we have to consider the following cases:

i) $W_{1}(x, y)=x y x y x y^{3}$,

ii) $W_{1}(x, y)=x y x y^{2} x y^{3}$,

iii) $W_{1}(x, y)=x y x y x y^{2}$.

In the case iii) we may introduce the relation $(x y)^{4}$ and get an infinite factor group because $\frac{1}{4}+\frac{1}{4} \geq \frac{1}{2}$.

i) Let $W_{1}(x, y)=x y x y x y^{3}$.

If $\gamma=2$ then we introduce the relation $y^{2}=1$ and get the infinite factor group $\bar{G}=\left\langle x, y, z \mid x^{2}=y^{2}=z^{2}=(x y)^{3}=(x z)^{2}=1\right\rangle$ because $n=2$ if $\gamma=2$.

Now, up to equivalence, let $\gamma=1$. We have already $\delta=\beta=1$.

If $n=2$ then $|G|=384$.

If $n=3$ then $|G|=9216$.

ii) Let $W_{1}(x, y)=x y x y^{2} x y^{3}$.

If $\gamma=2$, then we have $n=2$. Here we introduce the relation $y^{2}=1$ and get the infinite factor group $\bar{G}=\left\langle x, y, z \mid x^{2}=y^{2}=z^{2}=(x z)^{2}=1\right\rangle$.

Now, up to equivalence, let $\gamma=1$. We already have $\delta=\beta=1$. We introduce the relation $y^{2}=1$ and get the infinite factor group $\overline{\bar{G}}=\left\langle x, y, z \mid x^{2}=y^{2}=z^{n}=(y z)^{2}=(x z)^{2}=1\right\rangle$.

3.2.3 $k=4$ :

Again, let $\gamma \neq 2$ if $n=3$.

By [16], up to equivalence, we have to consider that following cases:

i) $W_{1}(x, y)=x y x y x y x y^{3}$,

ii) $W_{1}(x, y)=x y x y x y^{2} x y^{3}$,

iii) $W_{1}(x, y)=x y x y^{2} x y^{3} x y^{2}$.

Recall that $W_{1}(x, y)$ is not a proper power. In i) the trace polynomial of $W_{1}(x, y)$ is $t^{4}-2 t^{2}$, and 0 is a multiple root.

Hence $G$ is infinite by [18].

In both cases ii) and iii) we introduce the relation $y^{2}=1$ and get the infinite factor group $\bar{G}=\left\langle x, y, z \mid x^{2}=y^{2}=z^{n}=\left(y^{\gamma} z\right)^{2}=(x z)^{2}=1\right\rangle$ where, up to equivalence, $\gamma=1$ if $n=3$. 


\subsection{4 $k \geq 5$ :}

If $\rho: G \rightarrow \operatorname{PSL}(2, \mathbb{C})$ is an essential representation with $\rho(G)$ non-cyclic and finite then $\rho(G) \cong D_{8}$ or $S_{4}$, and hence the trace polynomial of $W_{1}(x, y)$ has a multiple root or $G_{1}$ has an essential representation $\rho_{1}: G_{1} \rightarrow \operatorname{PSL}(2, \mathbb{C})$ with $\rho_{1}\left(G_{1}\right)$ non-elementary. So, in any case, $G$ is infinite.

\subsection{Case $(1, \mathrm{~m})=(2,5)$}

We have $2 \leq n \leq 3$ because $\frac{1}{m}+\frac{1}{n}>\frac{1}{2}$. Then we may assume $\beta=\delta=1$ because $l=2$.

If $n=2$ then we also may assume that $\gamma=1$ by the dihedral equivalence. If $G_{1}$ has an essential representation $\rho_{1}: G_{1} \rightarrow \operatorname{PSL}(2, \mathbb{C})$ with $\rho_{1}\left(G_{1}\right)$ nonelementary then $G$ is infinite. If $G_{1}$ has an essential representation

$\rho_{1}: G_{1} \rightarrow \operatorname{PSL}(2, \mathbb{C})$ with $\rho_{1}\left(G_{1}\right)$ cyclic or infinite metabelian and $n \geq 3$ then $G$ is infinite by Fortsetzungssatz (2.5). If the trace polynomial of $W_{1}(x, y)$ has an multiple root then $G$ is infinite (see [18]).

\subsection{1 $k=2$ :}

Up to equivalence, we have to consider the following two cases:

i) $W_{1}(x, y)=x y x y^{2}$,

ii) $W_{1}(x, y)=x y x y^{3}$.

Let first $n=2$. Then, up to equivalence, $\gamma=\beta=\delta=1$. Also both cases are equivalent because of the dihedral move (replace $y$ by $y^{2}$ in case ii)). Now $|G|=240$.

Let now $n=3$. Then, up to equivalence, $G=\langle x, y, z| x^{2}=y^{5}=z^{3}=$ $\left.W_{1}^{2}(x, y)=\left(y^{\gamma} z\right)^{2}=(x z)^{2}=1\right\rangle$ with $1 \leq \gamma \leq 2$ and $W_{1}(x, y)=x y x y^{2}$ or $W_{1}(x, y)=x y x y^{3}$.

Let first $G=\left\langle x, y, z \mid x^{2}=y^{5}=z^{3}=\left(x y x y^{2}\right)^{2}=(y z)^{2}=(x z)^{2}=1\right\rangle$. Then $G$ is infinite. Here we used GAP and got the computational data:

$26[0,2,2,3]$ and

$$
\begin{array}{ccc}
x & \longmapsto & (3,6)(4,8)(5,11)(7,10)(9,14)(12,18)(13,16)(15,22)(17,23)(19,26) \\
& (20,21)(24,25), \\
y & \longmapsto & (1,2,4,9,8)(3,7,10,11,17)(5,6,12,19,24)(13,20,26,15,16) \\
(18,23,25,21,22), & \\
z & \longmapsto & (1,3,6)(2,5,11)(4,10,16)(7,8,13)(9,15,20)(12,17,24)(14,21,22) \\
& (18,25,23) .
\end{array}
$$

The group $G=\left\langle x, y, z \mid x^{2}=y^{5}=z^{3}=\left(x y x y^{3}\right)^{2}=\left(y^{2} z\right)^{2}=(x z)^{2}=1\right\rangle$ is equivalent to this first group and hence infinite (replace $y$ by $y^{2}$ ).

The last two groups are equivalent of order 14400.

\subsection{2 $k=3$ :}

Up to equivalence we have to consider the following cases: 
i) $W_{1}(x, y)=x y x y x y^{3}$,

ii) $W_{1}(x, y)=x y x y^{2} x y^{2}$,

iii) $W_{1}(x, y)=x y x y^{2} x y^{3}$,

iv) $W_{1}(x, y)=x y x y x y^{4}$ (see [16]).

We consider first the cases i) and ii).

Here $G_{1}$ has an essential representation $\rho_{1}: G_{1} \rightarrow \operatorname{PSL}(2, \mathbb{C})$ with $\rho_{1}\left(G_{1}\right)$ infinite metabelian. Hence, here $G$ is infinite if $n=3$.

Now, let $n=2$. Then the subgroup $H$ generated by $x$ and $y$ has a presentation

$$
H=\left\langle x, y \mid x^{2}=y^{5}=W_{1}^{2}(x, y)=W_{1}^{2}\left(x^{-1}, y^{-1}\right)=1\right\rangle \cong G_{1} .
$$

Therefore $G$ is infinite.

We now consider the cases iii) and iv).

If $n=2$ then, up to equivalence, we may assume that $\gamma=1$, and in both cases $G$ is finite of order 2400 .

Now, let $n=3$. Then, up to equivalence, $1 \leq \gamma \leq 2$. We have four groups to consider.

Let first $G=\left\langle x, y, z \mid x^{2}=y^{5}=z^{3}=\left(x y x y^{2} x y^{3}\right)^{2}=(y z)^{2}=(x z)^{2}=1\right\rangle$. Then $G$ is infinite. Here we used GAP and got the computational data:

$26[0,2,2,3]$ and

$$
\begin{array}{ccc}
x & \longmapsto & (3,6)(4,8)(5,11)(7,10)(9,14)(12,18)(13,16)(15,22)(17,23)(19,26) \\
& (20,21)(24,25), \\
y & \longmapsto & (1,2,4,9,8)(3,7,10,11,17)(5,6,12,19,24)(13,20,26,15,16) \\
& (18,23,25,21,22), \\
z & \longmapsto & (1,3,6)(2,5,11)(4,10,16)(7,8,13)(9,15,20)(12,17,24)(14,21,22) \\
& (18,25,23) .
\end{array}
$$

Let now $G=\left\langle x, y, z \mid x^{2}=y^{5}=z^{3}=\left(x y x y x y^{4}\right)^{2}=\left(y^{2} z\right)^{2}=(x z)^{2}=1\right\rangle$. Then $G$ is infinite. Again we used GAP and got the computational data:

$51[0,0,2,5]$ and

$$
\begin{array}{ccc}
x & \longmapsto & (1,2)(3,8)(4,11)(5,9)(7,18)(10,21)(13,23)(14,26)(15,28)(16,17) \\
& (19,22)(20,32)(24,25)(27,37)(29,39)(30,41)(33,44)(34,46) \\
& & (35,38)(36,45)(40,51)(42,43)(47,50)(48,49), \\
y & \longmapsto & (1,3,10,12,4)(2,6,8,19,7)(5,14,25,24,15)(11,22,31,18,21) \\
& (16,29,20,30,17)(23,33,43,42,34)(26,35,38,28,36) \\
& & (37,41,48,49,39)(44,47,50,46,51), \\
z & \longmapsto & (1,5,3)(2,8,9)(4,13,10)(6,16,17)(7,19,20)(11,21,23)(12,24,25) \\
& (14,27,15)(18,32,22)(26,28,37)(29,30,40)(31,42,43) \\
& (33,45,34)(35,47,48)(36,44,46)(38,49,50)(39,51,41) .
\end{array}
$$

The other two groups are finite of order 1728000 .

3.3.3 $k=4$ :

Here $G$ is infinite because $G_{1}$ always has an essential representation $\rho_{1}: G_{1} \rightarrow \operatorname{PSL}(2, \mathbb{C})$ with $\rho_{1}\left(G_{1}\right)$ non-elementary. 


\subsection{4 $k=5$ :}

We first consider the case $\alpha_{1}+\alpha_{2}+\alpha_{3}+\alpha_{4}+\alpha_{5} \equiv 0(\bmod 5)$. Then $G$ is infinite analogously to the cases i) and ii) in 3.3.2.

Now, let $\alpha_{1}+\alpha_{2}+\alpha_{3}+\alpha_{4}+\alpha_{5} \not \equiv 0(\bmod 5)$ Then, up to equivalence, we have to consider only $W_{1}(x, y)=x y x y^{3} x y^{2} x y^{4} x y^{\varepsilon}=: R_{\varepsilon}(x, y)$ with $1 \leq \varepsilon \leq 4$ (see [13]).

Also by [13], each of the $R_{\varepsilon}^{2}(x, y)=1$ is a consequence of each of the others, together with $x^{2}=1$ and $y^{5}=1$. Hence, it is enough to consider the case $\varepsilon=1$, that is

$W_{1}(x, y)=R_{1}(x, y)$. Also we may assume $1 \leq \gamma \leq 2$. Therefore we have to consider the case

$$
G=\left\langle x, y, z \mid x^{2}=y^{5}=z^{n}=\left(x y x y^{3} x y^{2} x y^{4} x y\right)^{2}=\left(y^{\gamma} z\right)^{2}=(x z)^{2}=1\right\rangle
$$

with $2 \leq n \leq 3$ and $1 \leq \gamma \leq 2$.

We consider $G_{1}=\left\langle x, y \mid x^{2}=y^{5}=\left(x y x y^{3} x y^{2} x y^{4} x y\right)^{2}=1\right\rangle$ and choose a slightly different essential representation $\rho_{1}: G_{1} \rightarrow \operatorname{PSL}(2, \mathbb{C})$ as usual. Let $X, Y \in \operatorname{PSL}(2, \mathbb{C})$ with $\operatorname{tr} X=0, \operatorname{tr} Y=\lambda-1$, where $\lambda=\frac{1}{2}+\frac{1}{2} \sqrt{5}=2 \cos \left(\frac{\pi}{5}\right)$, and $\tau:=\operatorname{tr} X Y$. Then $X$ has order 2 and $Y$ has order 5 in $\operatorname{PSL}(2, \mathbb{C})$. We get

$$
\operatorname{tr} X Y X Y^{3} X Y^{2} X Y^{4} X Y=(1-\lambda)(\lambda-1) \tau\left(\tau^{4}+(2-\lambda) \tau^{2}-1+\lambda\right) .
$$

Choose $\tau$ such that $\tau^{4}+(2-\lambda) \tau^{2}-1+\lambda=0$. Then we get an essential representation $\rho_{1}: G_{1} \rightarrow \operatorname{PSL}(2, \mathbb{C}), x \mapsto X, y \mapsto Y$, with $\rho_{1}\left(G_{1}\right)$ non-elementary. Hence, $G$ has a non-abelian free subgroup by Fortsetzungssatz (2.5), and especially $G$ is infinite.

In the following $\lambda$ always means $\lambda=\frac{1}{2}+\frac{1}{2} \sqrt{5}=2 \cos \left(\frac{\pi}{5}\right)$.

\subsection{5 $k \geq 6$ :}

If $\rho: G \rightarrow \operatorname{PSL}(2, \mathbb{C})$ is an essential representation with $\rho(G)$ non-cyclic and finite then $\rho(G) \cong D_{10}$ or $A_{5}$, and hence the trace polynomial of $W_{1}(x, y)$ has a multiple root or $G_{1}$ has an essential representation $\rho_{1}: G_{1} \rightarrow \operatorname{PSL}(2, \mathbb{C})$ with $\rho_{1}\left(G_{1}\right)$ non-elementary. So, in any case, $G$ is infinite.

\subsection{Case $(1, \mathrm{~m})=(2, \mathrm{~m}), \mathrm{m} \geq 6$}

Then $n=2$ because $\frac{1}{m}+\frac{1}{n}>\frac{1}{2}$. $G$ has now a factor group

$$
\bar{G}=\left\langle x, y, z \mid x^{2}=y^{m}=z^{2}=W_{1}^{2}(x, y)=(y z)^{2}=(x z)^{2}=1\right\rangle
$$

because $\left(y^{\beta} z\right)^{2}=1$ is a consequence of $(y z)^{2}=1$, together with $z^{2}=y^{m}=1$. If $\bar{G}$ is infinite then also $G$. The subgroup $H$ of $\bar{G}$ generated by $x$ and $y$ has index 2 and a presentation $H=\left\langle x, y \mid x^{2}=y^{m}=W_{1}^{2}(x, y)=W_{1}^{2}\left(x^{-1}, y^{-1}\right)=1\right\rangle$. If $G_{1}=\left\langle x, y \mid x^{2}=y^{m}=W_{1}^{2}(x, y)=1\right\rangle$ has an essential representation $\rho_{1}: G_{1} \rightarrow \operatorname{PSL}(2, \mathbb{C})$ with $\rho_{1}\left(G_{1}\right)$ cyclic or infinite metabelian then $H$ is infinite by $[6]$. 
Assume now that $G_{1}$ does not have such an essential representation. If $\rho: G \rightarrow \operatorname{PSL}(2, \mathbb{C})$ is an essential representation with $\rho(G)$ non-cyclic and finite then $\rho(G) \cong D_{2 m}$, and hence the trace polynomial of $W_{1}(x, y)$ has a multiple root 0 or $G_{1}$ has an essential representation $\rho_{1}: G_{1} \rightarrow \operatorname{PSL}(2, \mathbb{C})$ with $\rho_{1}\left(G_{1}\right)$ non-elementary.

So in any case, $G$ is infinite. This handles all cases with $l=2$. Hence, from now on let $l \geq 3$. Since $l \leq m$ and $\frac{1}{l}+\frac{1}{m}>\frac{1}{2}$ we get $l=3$.

\subsection{Case $(1, \mathrm{~m})=(3,3)$}

\subsection{1 $k=2$ :}

Then, up to equivalence, we have to consider the two cases:
i) $W_{1}(x, y)=x y x^{2} y^{2}$,
ii) $W_{1}(x, y)=x y x y^{2}$.

If $n=2$ then we may assume $\gamma=\delta=\alpha=\beta=1$, and get $|G|=576$ in case i) and $|G|=360$ in case ii).

Now, let $n=3$. We may assume $\delta=\beta=1$. Also, we may assume $\alpha=\gamma=1$ because $\left(x y^{2} x^{2} y\right)^{-1}=y^{2} x y x^{2} \sim x y x^{2} y^{2}$ and $x y^{2} x y \sim x y x y^{2}$ if we replace $y$ by $y^{2}$.

Now $|G|=11520$ in case i) and $|G|=7200$ in case ii).

Now, let $n=4$. Then $G$ is infinite if $W_{1}(x, y)=x y x y^{2}$. In this case the trace polynomial is $t^{2}-t-1$ with root $\lambda=2 \cos \left(\frac{\pi}{5}\right)$. But a finite subgroup of $\operatorname{PSL}(2, \mathbb{C})$ which is not cyclic and not dihedral cannot have an element of order 4 and the same time an element of order 5 .

Hence, let $W_{1}(x, y)=x y x^{2} y^{2}$.

If $\beta=2$ then $G_{3}=\left\langle x, z \mid x^{3}=z^{4}=\left(x z^{2}\right)^{2}=1\right\rangle$. Then $G_{3}$ has an essential representation $\rho_{3}: G_{3} \rightarrow \operatorname{PSL}(2, \mathbb{C})$ with $\rho\left(G_{3}\right)$ non-elementary, and hence $G$ is infinite by Fortsetzungssatz (the trace polynomial of $x z^{2}$ is $\sqrt{2} t-1$ which has the root $\left.\frac{1}{\sqrt{2}}\right)$.

Analogously, $G$ is infinite if $\delta=2$.

Now we may assume that $\beta=\delta=1$.

If $(\alpha, \gamma)=(2,1)$ then $G$ has the factor group

$$
\begin{aligned}
\bar{G} & =\left\langle x, y, z \quad \mid x^{3}=y^{3}=z^{4}=(x y)^{2}=(y z)^{2}=\left(x^{2} z\right)^{2}=1\right\rangle \\
& \cong\left\langle x, y, z \quad \mid x^{3}=y^{3}=z^{4}=\left(x y^{-1}\right)^{2}=\left(y z^{-1}\right)^{2}=\left(z x^{-1}\right)^{2}=1\right\rangle
\end{aligned}
$$

which is infinite by the Coxeter-citeria.

Analogously, $G$ is infinite if $(\alpha, \gamma)=(1,2)$.

The case $(\alpha, \gamma)=(2,2)$ is equivalent to the case $(\alpha, \gamma)=(1,1)$.

Hence, let $(\alpha, \gamma)=(1,1)$. Since $\left[x^{-1}, y\right] \sim[x, y]^{-1}$ we get that $G$ is againe infinite by the Coxeter-criteria.

Finally, let $n=5$. Let first $W_{1}(x, y)=x y x^{2} y^{2}=[x, y]$. Since $\left[x^{\varepsilon}, y^{\eta}\right] \sim[x, y]^{ \pm 1}$ for $\varepsilon= \pm 1, \eta= \pm 1$, we may assume that $\alpha=\beta=\gamma=1$. Then $G$ has the factor 
$\operatorname{group} \overline{\bar{G}}=\left\langle x, y, z \mid x^{3}=y^{3}=z^{5}=(x y)^{2}=\left(y z^{\delta}\right)^{2}=(x z)^{2}=1\right\rangle, 1 \leq \delta \leq 4$.

$\overline{\bar{G}}$ is infinite for $\delta=4$ by the Coxeter-criteria.

Again, since $\left[x^{-1}, y\right] \sim[x, y]^{-1}$ we get that $G$ is infinite also for $\delta=1$ by the Coxeter-criteria.

Now, let $2 \leq \delta \leq 3$.

Let first $\delta=3$. We choose $X, Y \in \operatorname{PSL}(2, \mathbb{C})$ with $\operatorname{tr} X=\operatorname{tr} Y=\operatorname{tr} X Y=1$. Now, using theorem 2.7 , we construct $Z \in \operatorname{PSL}(2, \mathbb{C})$ with $\operatorname{tr} Z=\lambda, \operatorname{tr} X Z=0$ and $\operatorname{tr} Y Z=1$. Then $Y Z^{3}$ has order 2 , and $x \mapsto X, y \mapsto Y, z \mapsto Z$ defines an essential representation $\rho: G \rightarrow \operatorname{PSL}(2, \mathbb{C})$ with non-real $\operatorname{tr} X Y Z$. Hence, $G$ is infinite if $\delta=3$.

If $\delta=2$ we replace $z$ by $z^{-1}$ and $x$ by $x^{-1}$. Since $\left[x^{-1}, y\right] \sim[x, y]^{-1}$ the group with $\delta=2$ is therefore equivalent to that with $\delta=3$, and $G$ is infinite also for $\delta=2$.

Now, let $W_{1}(x, y)=x y x y^{2}$. We may assume that $\alpha=\beta=\gamma=1$, that is

$$
G=\left\langle x, y, z \mid x^{3}=y^{3}=z^{5}=\left(x y x y^{2}\right)^{2}=\left(y z^{\delta}\right)^{2}=(x z)^{2}=1\right\rangle=: G(\delta)
$$

with $1 \leq \delta \leq 4 . G(\delta)$ is infinite for all $\delta$. First $G(1)$ and $G(4)$ are equivalent, and also $G(2)$ and $G(3)$ are equivalent.

Hence, we may assume that $1 \leq \delta \leq 2$. Here we used GAP und got the computational data:

1. $17[0,3,5]$ and

$$
\begin{aligned}
& x \longmapsto(3,7,8)(4,5,6)(9,12,10)(11,15,16)(13,17,14) \text {, } \\
& y \longmapsto(1,2,3)(4,10,9)(5,11,6)(12,13,14)(15,16,17) \text {, } \\
& z \longmapsto(1,4,2,6,5)(3,9,15,16,10)(7,12,8,14,13) \text {, }
\end{aligned}
$$

2. $17[0,3,5]$ and

$$
\begin{aligned}
& x \longmapsto(3,7,8)(4,5,6)(9,14,10)(11,13,12)(15,16,17) \text {, } \\
& y \longmapsto(1,2,3)(4,9,10)(5,6,11)(12,13,17)(14,15,16) \text {, } \\
& z \longmapsto(1,4,2,6,5)(7,8,13,17,12)(9,15,11,16,10), \\
& \text { for } \delta=2 \text {. }
\end{aligned}
$$

\subsection{2 $k=3$ :}

Up to equivalence, we here have only to consider the case $W_{1}(x, y)=x y x y x^{2} y^{2}$ (see [16]). If $n=2$ we may assume $\gamma=\delta=\alpha=\beta=1$ and get $|G|=2880$.

Now, let $n=3$. We may assume $\gamma=\beta=\alpha=1$ and $1 \leq \delta \leq 2$.

Let first $\delta=2$. The trace polynomial for $W_{1}(x, y)$ is $t(t-\lambda)(t-\lambda+1)$. We choose $X, Y \in \operatorname{PSL}(2, \mathbb{C})$ with $\operatorname{tr} X=\operatorname{tr} Y=1$ and $\operatorname{tr} X Y=\lambda$. Using 2.7 we construct $Z \in \operatorname{PSL}(2, \mathbb{C})$ with $\operatorname{tr} Z=1, \operatorname{tr} X Z=0$ and $\operatorname{tr} Y Z=1$. Then $Y Z^{2}$ has order 2 , and $x \mapsto X, y \mapsto Y, z \mapsto Z$ defines an essential representation $\rho: G \rightarrow \operatorname{PSL}(2, \mathbb{C})$ with non-real trace $X Y Z$. Hence, $G$ is infinite if $\delta=2$.

Now, let $\delta=1$. Then $G=\langle x, y, z| x^{3}=y^{3}=z^{3}=\left(x y x y x^{2} y^{2}\right)^{2}=(y z)^{2}=$ $\left.(x z)^{2}=1\right\rangle . G$ is infinite. Here we used GAP and got the computational data: $84[0,2,2]$ and 


$$
\begin{aligned}
& x \longmapsto(1,2,3)(4,10,11)(6,12,13)(7,15,16)(8,17,18)(9,19,20)(14,32,27) \\
& (21,40,41)(22,36,23)(24,43,44)(25,45,46)(26,49,47) \\
& (28,35,31)(29,53,54)(30,34,51)(33,56,42)(37,58,59) \\
& (38,60,61)(39,62,63)(48,71,68)(50,69,72)(52,70,55) \\
& (57,74,75)(64,79,73)(66,80,81)(67,82,83)(76,77,78) \text {, } \\
& y \longmapsto(1,4,5)(2,6,7)(3,8,9)(10,17,21)(11,23,24)(12,25,26)(13,29,30) \\
& (15,32,33)(16,31,34)(18,37,38)(20,22,39)(27,50,51) \\
& (28,52,47)(35,55,41)(36,57,42)(40,58,64)(43,56,53) \\
& (44,59,66)(45,67,62)(46,48,70)(49,71,60)(54,65,73) \\
& (61,68,77)(63,72,76)(69,74,84)(75,83,78)(79,82,80) \text {, } \\
& z \longmapsto(1,5,3)(4,9,8)(6,7,14)(10,18,22)(11,23,19)(12,27,28) \\
& (13,31,15)(16,35,32)(17,20,36)(21,42,37)(25,47,48) \\
& (26,46,50)(29,33,41)(30,55,34)(38,57,39)(40,59,53) \\
& (43,44,65)(45,68,69)(49,72,71)(51,70,52)(54,58,56) \\
& (60,63,76)(61,78,74)(62,75,77)(64,73,66)(67,84,83) \\
& (79,81,80) \text {. }
\end{aligned}
$$

If $n=4$ then $G$ is infinite because a non-cyclic, non-dihedral finite subgroup of $\operatorname{PSL}(2, \mathbb{C})$ cannot have an element of order 5 and the same time an element of order 4 (we may choose an essential representation $\rho: G \rightarrow \operatorname{PSL}(2, \mathbb{C})$ so that $\rho(x y)$ has order 5 and $\rho(z)$ has order 4$)$.

Now, let $n=5$. Then we may assume that $G=\langle x, y, z| x^{3}=y^{3}=z^{5}=$ $\left.\left(x y x y x^{2} y^{2}\right)^{2}=\left(y z^{\delta}\right)^{2}=(x z)^{2}=1\right\rangle$ with $1 \leq \delta \leq 4$.

If $\delta=4$ then we introduce the relation $(x y)^{2}=1$ and get the factor group $\bar{G}=\left\langle x, y, z \mid x^{3}=y^{3}=z^{5}=(x y)^{2}=\left(y z^{-1}\right)^{2}=(x z)^{2}=1\right\rangle$ which is infinite by the Coxeter-criteria ( $\bar{G}$ is an ordinary tetrahedron group).

Now, let $1 \leq \delta \leq 3$. $G$ is infinite. Here we used GAP and got the computational data:

1. $17[0,3,5]$ and

$$
\begin{array}{rll}
x & \longmapsto & (3,6,5)(4,8,11)(7,9,12)(10,13,16)(14,15,17), \\
y & \longmapsto & (1,2,4)(3,7,9)(5,6,10)(12,15,14)(13,16,17), \\
z & \longmapsto & (1,3,2,5,6)(4,9,13,16,7)(8,12,11,14,15) . \\
\text { if } \delta & =1, &
\end{array}
$$

2. $12[0,2,2]$ and

$$
\begin{aligned}
& x \longmapsto(1,2,3)(4,5,7)(6,9,10)(8,11,12) \text {, } \\
& y \longmapsto(1,2,4)(3,6,10)(5,8,7)(9,11,12) \text {, } \\
& z \longmapsto(1,3,2,5,7)(6,11,8,12,10) \text {. }
\end{aligned}
$$

3. $12[0,0,5]$ and

$$
\begin{array}{rll}
x & \longmapsto & (1,2,3)(4,5,7)(6,9,10)(8,11,12), \\
y & \longmapsto & (1,2,4)(3,6,10)(5,8,7)(9,11,12), \\
z & \longmapsto & (1,2,5,9,7)(4,6,11,12,10) . \\
\text { if } \delta & =3 . &
\end{array}
$$


3.5.3 $k=4$ :

Up to equivalence, by [16] we have to consider the following two cases:

i) $W_{1}(x, y)=x y^{-1} x^{-1} y^{-1} x y x^{-1} y$ and

ii) $W_{1}(x, y)=x^{-1} y^{-1} x y x y x^{-1} y$

(we here work with $x^{-1}, y^{-1}$ instead of $x^{2}, y^{2}$ ).

We first consider the case

i) $W_{1}(x, y)=x y^{-1} x^{-1} y^{-1} x y x^{-1} y$.

The trace polynomial of $W_{1}(x)$ is $t(t-1)(t-\lambda)(t-\lambda+1)$.

Let first $n=2$. Then, up to equivalence, $G=\langle x, y, z| x^{3}=y^{3}=z^{2}=$ $\left.\left(x y^{-1} x^{-1} y^{-1} x y x^{-1} y\right)^{2}=(y z)^{2}=(x z)^{2}=1\right\rangle . G$ is infinite. Here we used GAP and got the computational data:

$18[0,2,2,2]$ and

$$
\begin{aligned}
& x \longmapsto(1,2,4)(3,6,11)(5,8,13)(7,12,9)(10,15,14)(16,17,18) \text {, } \\
& y \longmapsto(1,3,7)(2,5,9)(4,6,10)(8,12,16)(11,14,17)(13,15,18) \text {, } \\
& z \longmapsto(2,4)(3,7)(5,10)(6,9)(8,14)(11,12)(13,15)(16,17) \text {. }
\end{aligned}
$$

This can be seen also as follows. Let $H$ be the subgroup of $G$ generated by $x$ and $y$.Then

$$
\begin{aligned}
H & =\langle x, y| x^{3}=y^{3}=\left(x y^{-1} x^{-1} y^{-1} x y x^{-1} y\right)^{2} \\
& \left.=\left(x^{-1} y x y x^{-1} y^{-1} x y^{-1}\right)^{2}=1\right\rangle \\
& =\left\langle x, y \mid x^{3}=y^{3}=\left(x y^{-1} x^{-1} y^{-1} x y x^{-1} y\right)^{2}=1\right\rangle
\end{aligned}
$$

because

$$
\begin{aligned}
\left(x^{-1} y x y x^{-1} y^{-1} x y^{-1}\right)^{-1} & =y x^{-1} y x y^{-1} x^{-1} y^{-1} x \\
& \sim x y^{-1} x^{-1} y^{-1} x y x^{-1} y .
\end{aligned}
$$

$H$ is infinite by [16].

Now, let $n=3$. Then, up to equivalence, $G=\langle x, y, z| x^{3}=y^{3}=z^{3}=$ $\left.\left(x y^{-1} x^{-1} y^{-1} x y x^{-1} y\right)^{2}=\left(y z^{\delta}\right)^{2}=(x z)^{2}=1\right\rangle$ with $1 \leq \delta \leq 2 . \quad G$ is infinite. Here we used also GAP and got the computational data:

1. $24[0,2,2,3]$ and

$$
\begin{array}{ccc}
x & \longmapsto & (1,2,3)(4,13,10)(5,12,16)(6,18,7)(8,9,11)(14,21,22) \\
(15,23,19)(17,24,20), & (1,2,4)(3,9,10)(5,15,17)(6,19,7)(8,13,14)(11,21,22) \\
y & \longmapsto & (12,23,18)(16,24,20), \\
& & (1,5,6)(2,7,8)(3,11,12)(4,14,15)(9,18,16)(10,20,21) \\
& & (13,19,17)(22,24,23),
\end{array}
$$

2. $24[0,2,2,3]$ and 


$$
\begin{aligned}
& x \longmapsto(1,2,3)(4,8,13)(5,12,16)(6,18,9)(7,11,10)(14,17,22) \\
& (15,23,20)(19,21,24) \text {, } \\
& y \longmapsto(1,3,4)(2,7,8)(5,17,12)(6,14,19)(9,22,16)(10,20,23) \\
& (11,13,15)(18,24,21) \text {, } \\
& z \longmapsto(1,5,6)(2,9,10)(3,11,12)(4,14,15)(7,18,16)(8,20,21)
\end{aligned}
$$

If $n=4$ then $G$ is certainly infinite analogously as in the earlier corresponding cases because $\lambda$ is a root of the trace polynomial.

Now, let $n=5$. Then, up to equivalence, $G=\langle x, y, z| x^{3}=y^{3}=z^{5}=$ $\left.\left(x y^{-1} x^{-1} y^{-1} x y x^{-1} y\right)^{2}=\left(y z^{\delta}\right)^{2}=(x z)^{2}=1\right\rangle$ with $1 \leq \delta \leq 4$. $G$ is infinite if $\delta=4$ (if we introduce the relation $(x y)^{2}=1$ then we get an infinite factor group by the Coxeter-criteria).

Now, let $\delta=3$. We choose $X, Y \in \operatorname{PSL}(2, \mathbb{C})$ with $\operatorname{tr} X=\operatorname{tr} Y=$ $\operatorname{tr} X Y=1$. Using theorem 2.7 we construct $Z \in \operatorname{PSL}(2, \mathbb{C})$ with

$\operatorname{tr} Z=\lambda, \operatorname{tr} X Z=0$ and $\operatorname{tr} Y Z=1$. Then $Y Z^{3}$ has order 2, and $x \mapsto X, y \mapsto Y, z \mapsto Z$ defines an essential representation

$\rho: G \rightarrow \operatorname{PSL}(2, \mathbb{C})$ with non-real trace $X Y Z$. Hence, $G$ is infinite for $\delta=3$.

If we replace $y$ by $y^{-1}$ we see that $G$ is also infinite if $\delta=1$ or $\delta=2$ because if we replace $y$ by $y^{-1}$ then $x y x^{-1} y x y^{-1} x^{-1} y^{-1} \sim x y^{-1} x^{-1} y^{-1} x y x^{-1} y$.

We now consider the case

ii) $W_{1}(x, y)=x^{-1} y^{-1} x y x y x^{-1} y$.

Here the trace polynomial of $W_{1}(x, y)$ is $(t-\lambda)(t-\lambda+1)(t+\lambda)(t+\lambda-1)$. If we choose $X, Y \in \operatorname{PSL}(2, \mathbb{C})$ with $\operatorname{tr} X=\operatorname{tr} Y=1$ and $\operatorname{tr} X Y=-\lambda$ then $x \mapsto X, y \mapsto Y$ defines an essential representation $\rho_{1}: G_{1} \rightarrow \operatorname{PSL}(2, \mathbb{C})$ with $\rho_{1}\left(G_{1}\right)$ a hyperbolic $(3,3,5)$-triangle group, and therefore especially non-elementary. Hence $G$ is infinite anyway. So, in fact the case ii) was superfluous but we have to mention it because in [16] the corresponding generalized triangle group $G_{1}$ was handled differently.

\subsection{4 $k \geq 5$ :}

If we assume that $G$ is finite then necessarily the trace polynomial of $W_{1}(x, y)$ must have a multiple root (the only possible roots for $G$ to be finite are $0,1, \lambda$ and $\lambda-1)$ which gives a contradiction. Hence, $G$ is infinite.

\subsection{Case $(1, \mathrm{~m})=(3,4)$}

Then $2 \leq n \leq 3$ because $\frac{1}{m}+\frac{1}{n}>\frac{1}{2}$.

3.6.1 $k=2$ :

Up to equivalence, by [19] we have to consider the cases: 
i) $W_{1}(x, y)=x y x^{2} y^{2}$,

ii) $W_{1}(x, y)=x y x^{2} y$,

iii) $W_{1}(x, y)=x y x y^{3}$,

iv) $W_{1}(x, y)=x y^{2} x^{2} y^{2}$.

Indeed ii) is superfluous but we have to mention it because in [19] the corresponding generalized triangle group was handled differently. So, if $W_{1}(x, y)=$ $x y x^{2} y$ then $G_{1}$ has an essential representation $\rho_{1}: G_{1} \rightarrow \operatorname{PSL}(2, \mathbb{C})$ with $\rho_{1}\left(G_{1}\right)$ non-elementary (the trace polynomial of $x y x^{2} y$ is $t^{2}-\sqrt{2} t-1$ ), and here $G$ is infinite.

Hence, we have to consider the cases i), iii) and iv).

Let first $n=2$. Then we may assume $\delta=\beta=\alpha=1$. If $\gamma=2$ then we introduce the relation $y^{2}=1$ and get an infinite factor group.

Hence, we may also assume that $\gamma=1$. Then $G=\langle x, y, z| x^{3}=y^{4}=z^{2}=$ $\left.W_{1}^{2}(x, y)=(y z)^{2}=(x z)^{2}=1\right\rangle$. The subgroup $H$ generated by $x$ and $y$ has a presentation $H=\left\langle x, y \mid x^{3}=y^{4}=W_{1}^{2}(x, y)=W_{1}^{2}\left(x^{-1}, y^{-1}\right)=1\right\rangle$.

If $W_{1}(x, y)=x y x^{2} y^{2}$ or $x y^{2} x^{2} y^{2}$ we introduce the relation $y^{2}=1$ and get the infinite factor group $C_{2} * C_{3}$.

If $W_{1}(x, y)=x y x y^{3}$ then $y W_{1}\left(x^{-1}, y^{-1}\right)^{-1} y^{-1}=W_{1}(x, y)$ and therefore $H=G_{1}$ which is infinite by [19].

Now, let $n=3$.

If $\gamma=2$ then $G_{2}=\left\langle y, z \mid y^{3}=z^{4}=\left(z^{2} y\right)^{2}=1\right\rangle$, and $G_{2}$ has an essential representation $\rho_{2}: G_{2} \rightarrow \operatorname{PSL}(2, \mathbb{C})$ with $\rho_{2}\left(G_{2}\right)$ non-elementary (the trace polynomial is $\sqrt{2} t-1)$.

Hence, let $\gamma \neq 2$. We may assume that $\gamma=1$. Then, up to equivalence, $G=\left\langle x, y, z \mid x^{3}=y^{4}=z^{3}=W_{1}^{2}(x, y)=(y z)^{2}=\left(x^{\alpha} z\right)^{2}=1\right\rangle$ with $1 \leq \alpha \leq 2$.

If $\alpha=2$ we introduce the relation $(x y)^{2}=1$ and get in each case an infinite factor group by the Coxeter-criteria.

Hence, let $\alpha=1$. If $W_{1}(x, y)=x y x^{2} y^{2}$ or $x y^{2} x^{2} y^{2}$ then again we introduce the relation $y^{2}=1$ and get an infinite factor group.

So, we are left with the group $G=\langle x, y, z| x^{3}=y^{4}=z^{3}=\left(x y x y^{3}\right)^{2}=(y z)^{2}=$ $\left.(x z)^{2}=1\right\rangle$. Here $G$ is infinite by GAP where we got the computational data:

$8[0,3]$ and

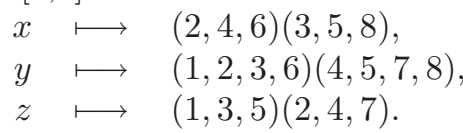

3.6.2 $k=3$ :

Up to equivalence, by [16] we have consider the following two cases:

i) $W_{1}(x, y)=x y x y x y^{3}$,

ii) $W_{1}(x, y)=x y x y x^{2} y^{3}$. 
The case i) is, analogously as above, superfluous because $G_{1}$ has a hyperbolic $(3,4,3)$-triangel group $\left\langle x, y \mid x^{3}=y^{4}=(x y)^{3}=1\right\rangle$ as a factor group (the trace polynomial is $(t-\sqrt{2})(t-1)(t+1)$, and we choose the root -1$)$. Analogously as above we have to mention this case.

Hence, let $W_{1}(x, y)=x y x y x^{2} y^{3}$. In this case the trace polynomial is $t^{2}(t-\sqrt{2})$ which has 0 as a multiple root. Hence, here also $G$ is infinite.

3.6.3 $k \geq 4$ :

If we assume that $G$ is finite then necessarily the trace polynomial of $W_{1}(x, y)$ must have a multiple root (the only possible roots for $G$ to be finite are 0,1 and $\sqrt{2}$ ) which gives a contradiction. Hence, $G$ is infinite.

\subsection{Case $(1, \mathrm{~m})=(3,5)$}

Then $2 \leq n \leq 3$ because $\frac{1}{m}+\frac{1}{n}>\frac{1}{2}$.

3.7.1 $k=2$ :

Up to equivalence, by [19] we have to consider the two cases:

i) $W_{1}(x, y)=x y x^{2} y^{2}$,

ii) $W_{1}(x, y)=x y x^{2} y^{3}$.

It is enough to consider the case i) because if we set $y=y^{\prime 2}$ and $x=x^{\prime 2}$ then $x y x^{2} y^{3}=x^{\prime 2} y^{\prime 2} x^{\prime} y^{\prime} \sim x^{\prime} y^{\prime} x^{\prime 2} y^{\prime 2}$.

Hence, let $W_{1}(x, y)=x y x^{2} y^{2}$. We may assume that $\delta=\beta=1$.

Let first $n=2$. Then, up to equivalence, $G=\langle x, y, z| x^{3}=y^{5}=z^{2}=$ $\left.\left(x y x^{2} y^{2}\right)=(x z)^{2}=(y z)^{2}=1\right\rangle$ and $|G|=43200$.

Now, let $n=3$. Up to equivalence, we have $G=\langle x, y, z| x^{3}=y^{5}=z^{3}=$ $\left.\left(x y x^{2} y^{2}\right)^{2}=\left(y^{\gamma} z\right)^{2}=(x z)^{2}=1\right\rangle$ with $1 \leq \gamma \leq 4$. If we set $y=y^{\prime 4}$ then $\left(x y^{\prime 4} x^{2} y^{\prime 3}\right)^{-1}=y^{\prime 2} x y^{\prime} x^{2} \sim x y^{\prime} x^{2} y^{\prime 2}$.

Hence, we may assume that $\gamma=2$ or $\gamma=4$.

If $\gamma=4$ then we introduce the relation $(x y)^{2}=1$ and get an infinite factor group by the Coxeter-criteria.

Now let $\gamma=2$, that is $G=\langle x, y, z| x^{3}=y^{5}=z^{3}=\left(x y x^{2} y^{2}\right)^{2}=\left(y^{2} z\right)^{2}=$ $\left.(x z)^{2}=1\right\rangle$. The trace polynomial of $x y x^{2} y^{2}$ is $\lambda t(t-\lambda)$. We choose

$X, Z \in \operatorname{PSL}(2, \mathbb{C})$ with $\operatorname{tr} X=\operatorname{tr} Z=1$ and $\operatorname{tr} X Z=0$. Using theorem 2.7 we construct $Y \in \operatorname{PSL}(2, \mathbb{C})$ with $\operatorname{tr} Y=\lambda, \operatorname{tr} X Y=\lambda$ and $\operatorname{tr} Y Z=\lambda-1$. Then $Y^{2} Z$ has order 2 , and $x \mapsto X, y \mapsto Y, z \mapsto Z$ defines an essential representation $\rho: G \rightarrow \operatorname{PSL}(2, \mathbb{C})$ with non-real trace $X Z Y$. Hence, $G$ is also infinite for $\gamma=2$.

3.7.2 $k=3:$

Up to equivalence, by [16] we have to consider the two cases: 
i) $W_{1}(x, y)=x^{-1} y x y^{-1} x y^{-1}$

ii) $W_{1}(x, y)=x^{-1} y x y^{3} x y^{-1}$.

The trace polynomial of $W_{1}(x, y)=x^{-1} y x y^{-1} x y^{-1}$ is

$f(t)=-(t-\lambda)(t-1)(t-\lambda+1)$, and the trace polynomial of $W_{1}(x, y)=x^{-1} y x y^{3} x y^{-1}$ is $f(t)=(t-1)^{2}(1-\lambda+1)$.

In case ii) the trace polynomial has 1 as a multiple root and, hence, $G$ is infinite in case ii) by [18].

Hence, let $W_{1}(x, y)=x^{-1} y x y^{-1} x y^{-1}$.

Let first $n=2$. Then, up to equivalence, $G=\langle x, y, z| x^{3}=y^{5}=z^{2}=$ $\left.W_{1}^{2}(x, y)=(y z)^{2}=(x z)^{2}=1\right\rangle$, and $|G|=1728000$.

Now, let $n=3$. Then, up to equivalence, $G=\langle x, y, z| x^{3}=y^{5}=z^{3}=$ $\left.W_{1}^{2}(x, y)=\left(z y^{\gamma}\right)^{2}=(x z)^{2}=1\right\rangle$ with $W_{1}(x, y)=x^{-1} y x y^{-1} x y^{-1}$ and $1 \leq \gamma \leq 4$. Let first $1 \leq \gamma \leq 3$. We choose $X, Z \in \operatorname{PSL}(2, \mathbb{C})$ with $\operatorname{tr} X=\operatorname{tr} Z=1$ and $\operatorname{tr} X Z=0$. Using theorem 2.7 we construct $Y \in \operatorname{PSL}(2, \mathbb{C})$ with $\operatorname{tr} Y=\lambda$, $\operatorname{tr} X Y=\lambda$ and

$$
\begin{aligned}
& \operatorname{tr} Y Z=0, \text { if } \gamma=1, \\
& \operatorname{tr} Y Z=\lambda-1, \text { if } \gamma=2, \\
& \operatorname{tr} Y Z=1, \text { if } \gamma=3 .
\end{aligned}
$$

Then $Y^{\gamma} Z$ has order 2 , and $x \mapsto X, y \mapsto Y, z \mapsto Z$ defines an essential representation $\rho: G \rightarrow \operatorname{PSL}(2, \mathbb{C})$ with non-real trace $X Z Y$. Hence, $G$ is infinite for $1 \leq \gamma \leq 3$

Now, let $\gamma=4$. Then $G$ is infinite by using GAP, and we got the computational data:

$12[0,2]$ and

$$
\begin{aligned}
& x \quad \longmapsto \quad(1,2,4)(3,5,7)(6,9,12)(8,11,10), \\
& y \quad \longmapsto \quad(2,4,8,12,11)(3,6,10,9,7), \\
& z \quad \longmapsto \quad(1,3,7)(2,5,4)(6,10,9)(8,12,11) .
\end{aligned}
$$

\subsection{3 $k=4$ :}

Up to equivalence, by [16] we have to consider the cases:

i) $W_{1}(x, y)=x^{-1} y x^{-1} y^{2} x y x y^{-1}=: R_{1}(x, y)$,

ii) $W_{1}(x, y)=x^{-1} y x y x^{-1} y^{2} x y^{-1}=: R_{2}(x, y)$,

iii) $W_{1}(x, y)=x^{-1} y x^{-1} y^{3} x y x y^{-1}=: R_{3}(x, y)$,

iv) $W_{1}(x, y)=x^{-1} y^{2} x y x^{-1} y x y^{-1}=: R_{4}(x, y)$.

If we replace $Y$ by $Y^{-1}$ then

$$
\left(R_{4}\left(x, y^{-1}\right)\right)^{-1}=y^{-1} x^{-1} y x y x^{-1} y^{2} \sim x^{-1} y x y x^{-1} y^{2} x y^{-1}=R_{2}(x, y) .
$$

Hence, it is not necessary to consider $R_{4}(x, y)$.

The trace polynomial of $R_{3}(x, y)$ is $f(t)=\lambda(t-1)(\lambda-t)\left(t^{2}-\lambda t+\lambda-1\right)-\lambda$, and 
therefore $G$ is infinite in case iii). In fact this case is superfluous but analogously as above we have to mention it.

The trace polynomial of $R_{1}(x, y)$ is $f(t)=\lambda t(t-1)(t-\lambda)(t-\lambda+1)$, and the trace polynomial of $R_{2}(x, y)$ is $f(t)=-\lambda t(t-1)(t-\lambda)(t-\lambda+1)$.

We consider in the following the cases i) and ii).

Let first $n=2$.

We start with the case ii). Then, up to equivalence, $G=\langle x, y, z| x^{3}=y^{5}=$ $\left.z^{2}=R_{2}^{2}(x, y)=(y z)^{2}=(x z)^{2}=1\right\rangle$.

Let $H$ be the subgroup generated by $x$ and $y$.

$H$ has a presentation $H=\left\langle x, y \mid x^{3}=y^{5}=R_{2}^{2}(x, y)=R_{2}^{2}\left(x^{-1}, y^{-1}\right)=1\right\rangle$. Let $K$ be the subgroup of $H$ generated by $x_{1}=y, x_{2}=x y x^{-1}$ and $x_{3}=x^{-1} y x$. $\mathrm{K}$ has a presentation

$$
\begin{aligned}
K=\left\langle x_{1}, x_{2}, x_{3}\right| x_{1}^{5}=x_{2}^{5}=x_{3}^{5} & =\left(x_{3} x_{1} x_{3}^{2} x_{1}^{-1}\right)^{2}=\left(x_{1} x_{2} x_{1}^{2} x_{2}^{-1}\right)^{2} \\
& =\left(x_{2} x_{3} x_{2}^{2} x_{3}^{-1}\right)^{2}=\left(x_{2}^{-1} x_{1}^{-1} x_{2}^{-2} x_{1}\right)^{2} \\
& \left.=\left(x_{3}^{-1} x_{2}^{-1} x_{3}^{-2} x_{2}\right)^{2}=\left(x_{1}^{-1} x_{3}^{-1} x_{1}^{-2} x_{3}\right)^{2}=1\right\rangle .
\end{aligned}
$$

The trace polynomial of $x_{3} x_{1} x_{3}^{2} x_{1}^{-1}$ is $-\lambda(t-\lambda)(t-1)$, and the trace polynomial of $x_{1}^{-1} x_{3}^{-1} x_{1}^{-2} x_{3}$ is also $-\lambda(t-\lambda)(t-1)$, ect.

We choose $X_{1}, X_{3} \in \operatorname{PSL}(2, \mathbb{C})$ with $\operatorname{tr} X_{1}=\operatorname{tr} X_{3}=\operatorname{tr} X_{1} X_{3}=\lambda$. Using theorem 2.7 we construct $X_{2}$ with $\operatorname{tr} X_{2}=\operatorname{tr} X_{1} X_{2}=\operatorname{tr} X_{3} X_{2}=\lambda$. Then $x_{1} \mapsto X_{1}$,

$x_{2} \mapsto X_{2}, x_{3} \mapsto X_{3}$ defines an essential representation $\rho: G \rightarrow \operatorname{PSL}(2, \mathbb{C})$ with non-real trace $X_{1} X_{2} X_{3}$. Hence, $G$ is infinite.

We now consider case i). Then, up to equivalence, $G=\langle x, y, z| x^{3}=y^{5}=z^{3}=$ $\left.R_{1}^{2}(x, y)=(y z)^{2}=(x z)^{2}=1\right\rangle$. Let $H$ be the subgroup generated by $x$ and $y$.

$H$ has a presentation $H=\left\langle x, y \mid x^{3}=y^{5}=R_{1}^{2}(x, y)=R_{1}^{2}\left(x^{-1}, y^{-1}\right)=1\right\rangle$. Let $K$ be the subgroup of $H$ generated by $x_{1}=y, x_{2}=x y x^{-1}$ and $x_{3}=x^{-1} y x$. $K$ has a presentation

$$
\begin{aligned}
K=\left\langle x_{1}, x_{2}, x_{3}\right| x_{1}^{5}=x_{2}^{5}=x_{3}^{5} & =\left(x_{3} x_{2}^{2} x_{3} x_{1}^{-1}\right)^{2}=\left(x_{1} x_{3}^{2} x_{1} x_{2}^{-1}\right)^{2} \\
& =\left(x_{2} x_{1}^{2} x_{2} x_{3}^{-1}\right)^{2}=\left(x_{2} x_{3}^{2} x_{2} x_{1}^{-1}\right)^{2} \\
& \left.=\left(x_{3} x_{1}^{2} x_{3} x_{2}^{-1}\right)^{2}=\left(x_{1} x_{2}^{2} x_{1} x_{3}^{-1}\right)^{2}=1\right\rangle .
\end{aligned}
$$

We choose $X_{1}, X_{2} \in \operatorname{PSL}(2, \mathbb{C})$ with $\operatorname{tr} X_{1}=\operatorname{tr} X_{2}=\operatorname{tr} X_{1} X_{2}=\lambda$. Using theorem 2.7 we construct $X_{3}$ with $\operatorname{tr} X_{3}=\operatorname{tr} X_{1} X_{3}=\operatorname{tr} X_{2} X_{3}=\lambda$. Then $\operatorname{tr} X_{i} X_{j}^{2} X_{i} X_{h}=0$ for all combinations with $1 \leq i, j, h \leq 3, h \neq i \neq j \neq h$. Hence, $x_{1} \mapsto X_{1}, x_{2} \mapsto X_{2}, x_{3} \mapsto X_{3}$ defines an essential representation $\rho: G \rightarrow \operatorname{PSL}(2, \mathbb{C})$ with non-real trace $X_{1} X_{2} X_{3}$. Hence, $G$ is infinite.

Let now $n=3$. We handle both cases simultaneously. Up to equivalence, $G=\left\langle x, y, z \mid x^{3}=y^{5}=z^{3}=R_{i}^{2}(x, y)=\left(z y^{\gamma}\right)^{2}=(x z)^{2}=1\right\rangle$ with $i=1$ or 2 and $1 \leq \gamma \leq 4$.

If $\gamma=4$ then we introduce the relation $(x y)^{2}=1$ and get an infinite factor group by the Coxeter-criteria. 
Let now $1 \leq \gamma \leq 3$. We choose $X, Z \in \operatorname{PSL}(2, \mathbb{C})$ with $\operatorname{tr} X=\operatorname{tr} Z=1$ and $\operatorname{tr} X Z=0$. Using theorem 2.7 we construct $Y \in \operatorname{PSL}(2, \mathbb{C})$ with $\operatorname{tr} Y=\lambda$, $\operatorname{tr} X Y=\lambda$ and

$$
\begin{aligned}
& \operatorname{tr} Y Z=0, \text { if } \gamma=1, \\
& \operatorname{tr} Y Z=\lambda-1, \text { if } \gamma=2, \\
& \operatorname{tr} Y Z=1, \text { if } \gamma=3 .
\end{aligned}
$$

Then the respective $Y^{\gamma} Z$ has order 2, and $x \mapsto X, y \mapsto Y, z \mapsto Z$ defines an essential representation $\rho: G \rightarrow \operatorname{PSL}(2, \mathbb{C})$ with non-real trace $X Z Y$. Hence, $G$ is infinite for $1 \leq \gamma \leq 3$, too.

\subsection{4 $k \geq 5$ :}

If we assume that $G$ is finite then necessarily the trace polynomial of $W_{1}(x, y)$ must have a multiple root (again, the only possible roots for $G$ to be finite are $0,1, \lambda$ and $\lambda-1$ ) which gives a contradiction. Hence, $G$ is infinite.

This proves the complete classification of the finite generalized tetrahedron groups.

Remark 3.5. There are unfortunately a few misprints in the paper [16] but only one is serious. On page 226 we showed that the generalized triangle group $G_{1}=\left\langle a, b \mid a^{3}=b^{5}=\left(a^{-1} b a b a^{-1} b^{2} a b^{-1}\right)^{2}=1\right\rangle$ has a free subgroup of rank 2. To do that, we considered the subgroup $H$ of $G$ generated by $x=b, y=a b a^{-1}$ and $z=a^{2} b a^{-2}$. $H$ has a presentation

$$
H=\left\langle x, y, z \mid x^{5}=y^{5}=z^{5}=\left(z x z^{2} x^{-1}\right)^{2}=\left(x y x^{2} y^{-1}\right)^{2}=\left(y z y^{2} z^{-1}\right)^{2}=1\right\rangle
$$

which is an generalized tetrahedron group.

$H$ has a free subgroup of rank 2 by theorem 2.1. So, the result is correct but the argument in [16] should be modified.

Let $H_{1}=\left\langle x, y \mid x^{5}=y^{5}=\left(x y x^{2} y^{-1}\right)^{2}=1\right\rangle$. It is possible to choose $\mu$ such that $\rho(x)=X=\left(\begin{array}{cc}0 & 1 \\ -1 & \lambda\end{array}\right), \rho(y)=Y=\left(\begin{array}{cc}\mu & -\mu+\lambda \mu-\mu^{2} \\ 1 & -1+\lambda-\mu\end{array}\right)$ extends to an essential, non-elementary representation of $H_{1}$ in $\operatorname{PSL}(2, \mathbb{C})$ with nonelementary image. In particular, $\langle X, Y\rangle$ has a free subgroup of rank 2, and so $G$ has one. 


\section{References}

[1] J. Cannon, D.F. Holt Computing maximal subgroups of finite index, J. Symbolic Comp. 37, (2004), 589-609.

[2] M. Conder, Three-relator quotients of the modular group, Quart. J. Math. Oxford (2)38 (1987), 427-447.

[3] H.S.M. Coxeter, Discrete groups generated by relections, Ann. Math. 35 (1934), 588-621.

[4] H.S.M. Coxeter, The complete enumeration of finite groups form the from $R_{i}^{2}=\left(R_{i} R_{j}\right)^{k_{i j}}, \mathrm{~J}$. London Math. Soe. 10 (1935), 21-25.

[5] M. Edjvet, J. Howie, G. Rosenberger, T. Thomas, Finite generalized tetrahedron groups with a high-power relator, Geometriae Dedicata 94, (2002), 111-139.

[6] M. Edjvet, G. Rosenberger, M. Stille, R. Thomas, On certain finite generalized tetrahedron groups: Computational and Geometric Aspects of Modern Algebra, London Math. Soe. Lecture Note Series 275, Cambridge University Press (2000), 54-65.

[7] B. Eick, D.F. Holt, E.A. O'Brien, Handbook of computational Group Theory, Discrete Mathematics and its Applications, Chapmann and Hall/CRC, Boca Raton, FL, (2005).

[8] B. Eick, A. Hulpke, Computing the maximal subgroups of a permutation group I: Groups and Computation III, (Columbus, Ohio, (1999)), Ohio State Univ. Math. Res. Inst. Publ. 8, de Gruyter, Berlin (2001), 155-168.

[9] B. Fine, F. Levin, F. Roehl, G. Rosenberger, The generalized tetrahedron groups: Geometric Group Theory, (Columbus, Ohio (1992)), Ohio State University Math. Results Inst. Publ. 3, de Gruyter, Berlin (1995), 99-119.

[10] B. Fine, G. Rosenberger, Algebraic Generalizations of Discrete Groups, Monographs and Textbooks in Pure and applied Math. 223, Marcel Dekker Inc., New York (1999).

[11] B. Fine, A. Hulpke, V. gr. Rebel, G. Rosenberger The Tits alternative for Spherical Generalized Tetrahedron Groups, Algebra Colloquium, to appear.

[12] GAP-Groups, Algorithms Programming - a System for Computational Discrete Algebra, Version 4.4.9 (2006), (www.gap-system.org).

[13] J. Howie, V. Metaftsis, R. Thomas, Finite Generalized Triangle Groups, Trans. AMS 347 (1995), 3613-3623.

[14] J. Howie, N. Kopteva, The Tits Alternative for generalized tetrahedron groups, J. Group Theory 9 (2006), 173-189. 
[15] A. Hulpke, Representing subgroups of finitely presented groups by quotient subgroups, Experiment. Math. 3 (2001), 369-381.

[16] F. Levin, G. Rosenberger, On free subgroups of generalized triangle groups: Part II Group Theory, World Scientific (1992), 206-228.

[17] G. Rosenberger, M. Scheer, R. Thomas, Finite generalized tetrahedron groups with a cubic relator: Groups, St. Andrews 2001, Oxfort, Vol. II, London Math. Soe. Lecture Note Ser. 305, Cambridge Univ. Press (2003), $455-486$.

[18] G. Rosenberger, M. Scheer Classification of the finite generalized tetrahedron groups, Contemporary Math. 296 (2002), 207-229.

[19] G. Rosenberger, On free subgroups of generalized triangle groups, Algebra i Logika 228 (1989), 227-240.

[20] C. Runge, Endliche Tetraedergruppen - Der Tsaranov-Fall, Diplomarbeit, Dortmund (1998).

[21] S.V. Tsaranov, Finite generalized Coxeter groups, Algebras, Groups and Geometries 6 (1989), 281-318.

[22] S.V. Tsaranov, Finite generalized Coxeter groups, Algebras, Groups and Geometries 6 (1989), 421-457. 


\section{Benjamin Fine}

Department of Mathematics

Fairfield University

Fairfield, CT 06430, USA

fine@mail.fairfield.edu

\section{Miriam Hahn}

TU Dortmund

Department of Mathematics

44221 Dortmund, Germany

miriam.hahn@tu-dortmund.de

Alexander Hulpke

Department of Mathematics

Colorado State University

Fort Collins, CO 80523, USA

hulpke@math.colostate.edu

Volkmar große Rebel

TU Dortmund

Department of Mathematics

44221 Dortmund, Germany

volkmar.rebel@mathematik.uni-dortmund.de

Gerhard Rosenberger

TU Dortmund

Department of Mathematics

44221 Dortmund, Germany

Gerhard.Rosenberger@math.uni-dortmund.de

Martin Scheer

TU Dortmund

Department of Mathematics

44221 Dortmund, Germany

Martin.Scheer@mathematik.uni-dortmund.de 